\title{
Lopsided Galaxies, Weak Interactions and Boosting the Star Formation Rate
}

\author{
Gregory Rudnick, Hans-Walter Rix 四, Robert C. Kennicutt, Jr. \\ Steward Observatory, University of Arizona, Tucson AZ 85721
}

\begin{abstract}
To investigate the link between weak tidal interactions in disk galaxies and the boosting of their recent star formation, we obtain images and spatially integrated spectra (3615 $\AA \leq \lambda \leq 5315 \AA$ ) for 40 late-type spiral galaxies (Sab-Sbc) with varying degrees of lopsidedness (a dynamical indicator of weak interactions). We quantify lopsidedness as the amplitude $\left\langle\tilde{A}_{1}\right\rangle$, of the $m=1$ Fourier component of the azimuthal surface brightness distribution, averaged over a range of radii. The median spectrum of the most lopsided galaxies shows strong evidence for a more prominent young stellar population (i.e. strong Balmer absorption, strong nebular emission, a weak $4000 \AA$ break and a blue continuum) when compared to the median spectrum of the most symmetric galaxies. We compare the young stellar content, quantified by $E W\left(H \delta_{a b s}\right)$ and the strength of the $4000 \AA$ break $\left(D_{4000}\right)$, with lopsidedness and find a $3-4 \sigma$ correlation between the two. We also find a $3.2 \sigma$ correlation between $E W\left(H \beta_{\text {emission }}\right)$ and lopsidedness. Using the evolutionary population synthesis code of Bruzual \& Charlot we model the spectra as an "underlying population" and a superimposed "boost population" with the aim of constraining the fractional boost in the SFR averaged over the past 0.5 Gyr (the characteristic lifetime of lopsidedness). From the difference in both $E W\left(H \delta_{a b s}\right)$ and the strength of the $4000 \AA$ break $\left(D_{4000}\right)$ between the most and least symmetric thirds of our sample, we infer that $\sim 1 \times 10^{9} M_{\odot}$ of stars are formed over the duration of a lopsided event in addition to the "underlying" SFH (assuming a final galactic stellar mass of $10^{10} M_{\odot}$ ). This corresponds to a factor of 8 increase in the SFR over the past $5 \times 10^{8}$ years. For the nuclear spectra, all of the above correlations except $D_{4000}$ vs. $\left\langle\tilde{A}_{1}\right\rangle$ are weaker than for the disk, indicating that in lopsided galaxies, the SF boost is not dominated by the nucleus.
\end{abstract}

\footnotetext{
${ }^{1}$ Alfred P. Sloan Fellow

${ }^{2}$ Current address Max-Planck-Institut für Astronomie, Heidelberg, Germany
} 
Subject headings: galaxies: evolution — galaxies: interaction — galaxies:

kinematics and dynamics — galaxies: spiral — galaxies: structure — stars: formation

\section{INTRODUCTION}

Galaxies do not live isolated lives, but exist in the tidal fields of their environment. Arp (1966), in his Atlas of Peculiar Galaxies, lay the observational groundwork for the modern study of interacting galaxy systems by identifying many "peculiar" systems, later interpreted as various stages of major galaxy mergers. Strong galaxy-galaxy interactions may dramatically alter the stellar populations (e.g. Larson \& Tinsley 1978; Kennicutt et al. 1987; Turner 1998; Kennicutt 1998), morphology (e.g. Toomre \& Toomre 1972; Hernquist, Heyl \& Spergel 1993) and kinematics of galaxies (e.g. Toomre \& Toomre 1972; Barnes \& Hernquist 1992) driving evolution along the Hubble sequence. Massive mergers are also capable of funneling gas into the center of galaxies causing nuclear starbursts (Barnes \& Hernquist 1991; Mihos, Richstone \& Bothun 1992; Barnes \& Hernquist 1996) and QSO activity (e.g. Sanders et al. 1988). At the present epoch, however, major mergers are fairly rare events (e.g. Kennicutt et al. 1987) and their broad evolutionary importance is unclear.

Minor mergers and, in general, weak tidal interactions between galaxies occur with much higher frequency than major ones (e.g. Lacey \& Cole 1993). By weak interactions we mean those which do not destroy the disk of the "target" spiral. Hierarchical structure formation models (e.g. cold dark matter) predict that the merging histories for high mass objects today contained multiple low mass accretion events in their past (e.g. Lacey \& Cole 1993). The specific roles which weak interactions play in the evolution of galaxies, however, is uncertain. Weak interactions may cause disk heating (e.g. Toth \& Ostriker 1992; Quinn, Hernquist \& Fullagar 1993) and satellite remnants may build up the stellar halo (e.g. Searle \& Zinn 1978; Johnston, Hernquist \& Bolte 1996). Kennicutt et al. (1987) studied the relation between interaction strength and star formation by making a comparison between isolated galaxies, close pairs, and galaxies from the Arp Atlas. They found that close pairs have larger values of $E W\left(H \alpha_{e m}\right)$, i.e. higher star formation rates (SFR) than isolated galaxies. While pair spacing is weakly correlated with the SFR, they could not determine the specific role of interaction strength on the SFR. Hashimoto et al. (1998) and Allam et al. (1999) both studied the Hubble type specific effects of environment on the SFR in galaxies. They found that the SFR/mass of existing stars was inversely proportional to the local galaxy density. They postulate that the anti-correlation is due partly to gas stripping and due partly to the anti-correlation of the merger cross-section with the galaxy-galaxy 
velocity dispersion.

There is also evidence that interactions excite nuclear activity. In their close pair and strongly interacting sample Kennicutt et al. (1987) found a strong correlation between $H \alpha$ emission in the disk and that in the nucleus. Such a correlation between disk and nuclear emission is supported by theoretical work; Mihos \& Hernquist (1994) and Hernquist \& Mihos (1995) demonstrated that minor interactions form bar instabilities in the disk which in turn funnel large amounts of gas into the nucleus. The effectiveness of this process is suppressed by the presence of a dense bulge, which prevents bar formation. Due to the numerical expense in computing high resolution N-body/SPH (collisionless particle/smoothed particle hydrodynamics) models, the exact interaction parameters which result in such activity are uncertain.

Weak interactions may also manifest themselves as kinematic or structural irregularities. Roughly $50 \%$ of all spiral galaxies have asymmetric HI profiles and rotation curves (Baldwin, Lynden-Bell \& Sancisi 1980; Richter \& Sancisi 1994; Haynes et al. 1998). Baldwin et al. (1980) postulated that these asymmetries are caused by weak interactions in the galaxy's past or by lopsided orbits. Barton et al. (1999) examined the optical rotation curves of a set of observed and simulated interacting disk galaxies. They showed that interactions can cause large scale, time dependent asymmetries in the rotation curves of their sample galaxies. Swaters et al. (1999) studied the kinematic asymmetries present in two galaxies lopsided in their optical and HI distributions. They qualitatively reproduced the kinematic asymmetries by placing closed orbits in mildly lopsided potential.

A dynamical indicator of weak interactions may be "lopsidedness." In the context of this paper (following Rudnick \& Rix 1998; hereafter RR98), lopsidedness is defined as a bulk asymmetry in the mass distribution of a galactic disk. Surveys for lopsidedness in the stellar light of galaxies were first carried out by Rix \& Zaritsky (1995; hereafter RZ95) and Zaritsky \& Rix (1997; hereafter ZR97). Using near-IR surface photometry of face-on spiral galaxies (spanning all Hubble types) they examined the magnitude of the $m=1$ azimuthal Fourier component of the I and K-band surface brightness, thus characterizing the global asymmetry of the stellar light. RZ95 and ZR97 found that a quarter of the galaxies in their sample were significantly lopsided. Using a larger, magnitude limited sample restricted to early type disks ( $\mathrm{S} 0$ to Sab) and imaged in the R-band, RR98 found that the fraction of significantly lopsided early type disks is identical to that for late-type disks. RR98 convincingly demonstrated that lopsidedness is not an effect of dust, but is in fact the asymmetric distribution of the light from old stars and hence from the stellar mass in the disk.

Some theoretical work has been done in examining long lived $m=1$ modes (Syer \& 
Tremaine 1996; Zang \& Hohl 1978; Sellwood \& Merritt 1994), little convincing evidence however has been put forth to show that isolated galaxies will form stable $m=1$ modes without external perturbations or significant counter-rotating populations. Without invoking the special cases above, long lived lopsidedness is possible if the disk resides in a lopsided potential. The question remains however: how is a lopsided potential created/maintained? Numerical simulations of hyperbolic encounters between disk galaxies fail to produce $m=1$ modes of amplitude $>10 \%$ without destroying the pre-existing stellar disk (Naab, T.; private communication). Minor mergers and possibly some weak interactions therefore remain as the most probable cause of lopsidedness (RR98). Recent work has shown that perturbations in the outer halo of a galaxy may be amplified and even transmitted down into the disk (Weinberg 1994). Work by Walker, Mihos \& Hernquist (1996) and ZR97 showed that the type and magnitude of lopsidedness seen in RZ95, ZR97 and RR98 is comparable to the result of the accretion of a small satellite, if the mass ratio with the main galaxy is $\approx 1 / 10$. In a preliminary study (i.e. a rigid halo with no dynamical friction) Levine and Sparke (1998) showed that lopsided galaxies may be formed by disks orbiting off center and retrograde in a flat-cored, dark matter dominated halo. They postulated that a galaxy may be pushed off center by a satellite accretion.

Using phase mixing arguments (Baldwin et al. 1980; RZ95) and analysis of N-body simulations (Walker et al. 1996; ZR97) the lifetime of lopsided features has been estimated at $t_{\text {lop }} \approx 1$ Gyr. That lopsidedness is transient $\left(t_{\text {lop }} \ll t_{\text {Hubble }}\right)$ yet common, requires that it must be recurring and therefore lopsidedness may have significant evolutionary consequences.

The current paper focuses on the impact that minor mergers (observed as lopsidedness) may have on boosting the SFR and the recent star formation history (SFH) of disk galaxies. For the purpose of this discussion, we will assume that lopsidedness is caused by minor mergers. Regardless of what causes lopsidedness however, the perturbation in the gravitational potential manifestly exists and therefore may affect the gas in the galaxy to such a degree as to boost the SFR. Indeed, ZR97 find that lopsidedness is correlated (at $\geq 96 \%$ confidence) with the "excess" of blue luminosity (over what is predicted by the Tully-Fisher relation). Modeling the integrated spectral evolution of starbursts using evolutionary population synthesis (EPS) codes has been been well studied (e.g. Couch and Sharples 1987; Barger et al. 1996; Turner 1998) and despite its limitations, is a useful tool in determining the relative SFH over the past 1 Gyr. The same techniques used to probe the SFH in massive starbursts should also work to probe the recent SFH in the putative mini-bursts which we seek to study. By comparing measured indicators of recent SF (e.g. $E W\left(H \delta_{a b s}\right), 4000 \AA$ break strength, A star content), to the same indicators derived from the EPS models, we will place limits on the mini-burst mass and duration. 
We have obtained spatially integrated spectra of a sample of 40 late type spiral galaxies (Sab-Sbc) of varying degrees of lopsidedness with the intent of using their relative stellar populations (as determined from stellar template fitting and EPS models) to determine their recent SF histories. Unlike the mass-normalized blue light excess, $\Delta B$ used in ZR97, our method operates independently of assumptions about a galaxy's mass, inclination or luminosity. In addition to probing the recent ( $\leq 1 \mathrm{Gyr}$ ) SFH with studies of the stellar continuum we probe the current SFR by measuring the integrated Balmer line emission strengths (e.g. Kennicutt et al. 1994).

The layout of the paper is as follows. In $\S 2$ we discuss the sample selection, observations, data reduction and determination of galaxy lopsidedness; In $\S 3$ we examine our methods for determining the current SFR and recent SFH via the measurement of emission and stellar continuum properties as a function of lopsidedness. The discussion of the significance of these results, including the correlation of the boost parameters with other galaxy characteristics and the impact of our results on previous works (i.e. RZ95,ZR97 \& RR98) is contained in $\S 4$. In $\S 5$ we present a summary and possible directions for future work.

\section{THE DATA}

\subsection{Sample Selection}

To build a sample of galaxies with varying degrees of lopsidedness, we imaged a large number of galaxies $\left(N_{\text {gal }} \geq 100\right)$ taken from the RC3 catalog (De Vaucouleurs et al. 1991), selected according to the following criteria: apparent blue magnitude $\mathrm{m}_{B} \leq 14$, redshift $\mathrm{cz} \leq 10,000 \mathrm{~km} / \mathrm{s}$, axis ratio $b / a \geq 0.64\left(50^{\circ} \leq i \leq 0^{\circ}\right)$, de Vaucouleurs type $a b \rightarrow b c$, and a maximum diameter of $4^{\prime}$. The median diameter of the galaxies in our sample was $1.8^{\prime}$. The magnitude and redshift limits were chosen to minimize the required exposure times. The axis ratio of the galaxies was constrained because it is hard to measure an azimuthal

$m=1$ component in a highly inclined galaxy. Once imaging was obtained and lopsidedness determined for each galaxy (see §2.2.1), we constructed a sample for spectroscopy consisting of 40 of our imaged galaxies (see Table 1). These were selected to give the sample equal numbers of lopsided and symmetric targets.

Our sample is partially selected according to Hubble type, and we must explore the effects which morphological evolution induced by lopsidedness may have on our conclusions. Walker et al. (1996) suggested that minor mergers increase bulge size, heat the galactic disk vertically and consume a large fraction of the galaxy's gas, eventually resulting in a low post-merger SFR. These two effects may drive galaxies towards earlier Hubble type 
after they experience minor mergers. Evolutionary processes such as these however become dominant either after the expected lifetime of lopsidedness $(t \geq 1 \mathrm{Gyr})$, or after repeated merger events (Walker et al. 1996). During the interaction itself the irregularity in structure made manifest by lopsidedness as well as the creation of spiral arms via tidal perturbations will temporarily move a galaxy later in Hubble type. This will effectively push lopsided early type disk galaxies into our sample while pushing those of later type out of it. Due to their lower gas masses (Roberts \& Haynes 1994), early type spirals have less potential for a large absolute increase in their SFR than late types. Small boosts, however, may be easily noticeable against the typically older stellar population of early type disks. The exact interplay of these two effects may bias our measurement of the relation between SFR and lopsidedness.

\subsection{Observations}

\subsubsection{Imaging}

Our imaging data were obtained during runs at Steward Observatory's 2.3-m Bok reflector on Kitt Peak (1997 November 6-7 and 1998 February 1-2) and at its 61-inch (1.5-m) reflector on Mt. Bigelow (1998 May 15-18). The CCD pixel scales at the Bok reflector and Bigelow reflector were $0^{\prime \prime} .4$ pixel $^{-1}$ with fields of view $6.8 \times 66^{\prime} .8$ and $33^{\prime} 4 \times 33^{\prime} 4$ respectively. The median seeing at the 2.3-m for the November and February runs were 1".3 and 1 ".5, respectively, while the median seeing at the $1.5-\mathrm{m}$ in May was 1 1"3.

A Nearly-Mould $R$-band filter $\left(\lambda_{\text {center }}=650 \mathrm{~nm}\right)$ was used at the $2.3-\mathrm{m}$ and a Kron-Cousins R-band filter with $\lambda_{\text {center }}=650 \mathrm{~nm}$ was used at the $1.5-\mathrm{m}$. The effects of wavelength on observed lopsidedness are discussed in $\S 2.1$ of RR98, and found not to be critical.

To determine the lopsidedness of a galaxy we perform an azimuthal Fourier decomposition of the R-band surface brightness, as in RR98:

$$
I\left(R_{m}, \phi\right)=a_{o}\left\{1+\sum_{j=1}^{N} a_{j} e^{-i\left[j\left(\phi_{j}-\phi_{j}^{o}\right)\right]}\right\},
$$

where for each radius, $\left|a_{o}\right|(R)$ is the average intensity and $\left|a_{1}\right|(R)$ describes the lopsidedness. We define the luminosity normalized quantities $A_{1} \equiv a_{1} / a_{0}$. Instead of $A_{1}(R)$, we use $\tilde{A}_{1}(R)$ (the error corrected value which accounts for the positive definite nature of our measurements and the presence of errors; see RR98 for details) as our measure of asymmetry. We calculate the mean asymmetry of each galaxy, $\left\langle\tilde{A}_{1}\right\rangle$ (see Table 2), from 1.5 to 2.5 disk scale lengths using the weighted average described in RR98. 


\subsubsection{Spectroscopy}

Spectra were obtained with the Bollers \& Chivens Spectrograph at the 2.3-m Bok reflector during the nights, 1998 March 22-25, 1998 May 25-28, and 1998 June 29. We used a $400 \mathrm{gmm}^{-1}$ in $2^{\text {nd }}$ order, blazed at $3753 \AA$, and a $2.5^{\prime \prime}$ slit, resulting in a resolution of $\approx 1500$ and a wavelength range of $3600 \AA \lesssim \lambda \lesssim 5300 \AA$. This range includes the entire Balmer series redward to $H \beta$, the $4000 \AA$ break, $\mathrm{Ca} \mathrm{H}+\mathrm{K}$ doublet, [O II] $] \lambda \lambda 3726,3729 \AA$ and [O III $] \lambda \lambda 4959,5007 \AA$. To reduce read noise, the CCD was binned in the spatial direction; on 22 March we binned by 2 for a resultant pixel scale of $1.67^{\prime \prime}$ pixel $^{-1}$ while on all other nights we binned by 4 for a pixel scale of $3.33^{\prime \prime}$ pixel $^{-1}$. A $\mathrm{CuSO}_{4}$ filter was used to block $1^{\text {st }}$ order light. Aside from using standard stars to calibrate the relative spectral response of the instrument, no absolute flux calibration was attempted. This was done partly because of the non-photometric conditions of some of our nights and partly due to the independence of our analysis methods on absolute flux levels.

Following Kennicutt (1992), we obtained spatially integrated spectra of the galaxies by repeatedly scanning the slit across the galaxy between $-2.5 R_{\exp } \leq x \leq 2.5 R_{\text {exp }}$. For each galaxy we obtained 2 exposures of 25 minutes each. To isolate the disk contributions to the integrated spectra, we also obtained a 5 minute exposure of the nucleus for each galaxy for later subtraction.

\subsection{Reduction}

\subsubsection{Images}

The basic image reduction was carried out with standard IRAF 1 routines. The total dark current was $\leq 1 \mathrm{e}^{-} /$exposure/pixel and so it was ignored. The images were flat-fielded with a combination of twilight and smoothed night sky flats. High $S / N$ twilight flats $(N \approx 5)$ were used to take out small-scale variations and the lower $S / N$ smoothed night sky flats $(N \approx 6)$ were used to take out large-scale sensitivity fluctuations. The large-scale flat-field quality was determined by measuring variance in the median sky level at the four corners of the images. In all images where the galaxy was small in the field of view, the images were found to be flattened to better than $1 \%$. In some cases, the large size of the galaxies in comparison to the field of view at the $1.5-\mathrm{m}$ telescope precluded such an estimate.

\footnotetext{
${ }^{3}$ IRAF is distributed by the National Optical Astronomical Observatories, which are operated by AURA, Inc. under contract to the NSF.
} 
Point sources in the images were selected using DAOFIND, and surrounding pixels were excised in the subsequent analysis to a radius where the stellar point spread function declined to the level of the sky.

\subsubsection{Spectra}

The basic spectral reduction was carried out with IRAF routines. The measured dark current from multiple 25 minute dark exposures was $\leq 2 \mathrm{e}^{-} / \exp /$ pixel and so was not accounted for. Small-scale variations were removed with a combined series of 2 min. dome flats. Twilight flats were fit in the spatial direction with a $5-7^{\text {th }}$ order cubic spline at several different positions in the spectral direction to construct a map of the slit response as a function of wavelength. Non-linear pixels were interpolated over using a bad pixel mask generated from the ratio between a combined series of 2 minute and 10 second dome flats.

Removing cosmic rays from spectra without accidently removing emission lines is best automated by using the shape of the spectrum itself in the cleaning process: for a given

column (spatial direction), we medianed together the target column with the eight columns on either side to construct a slit profile, $I_{\text {slit }}$. Using $\chi^{2}$ minimization, we fit the target column with the the following function:

$$
I_{\text {model }}(y)=a_{1} I_{\text {slit }}(y)+a_{2} y+a_{3}
$$

where the $a_{3}$ term accounts for the sky background. Pixels deviating by more than $7 \sigma$ from the best fit are replaced with the value of the best fit model at that point. Flagged segments larger than the spectral resolution of the instrument were not removed so as to avoid the accidental cleaning of emission lines. This algorithm is very efficient and, once a suitable set of parameters (i.e. $N_{\text {med }}$, threshold) has been chosen, can remove almost all of the cosmic rays on the chip.

After cosmic ray removal, the spectra were rectified using He-Ar lamps taken at various times during the night. Background contributions were then subtracted. To extract the spectra, we summed the number of center rows which corresponded to $\pm 2.5 R_{\text {exp }}$ for the disk spectra and extracted the center row only for the nuclear spectra. Using standard stars taken during the night, we then removed the spectral response of the system from the 1D, extracted spectra. Finally, the wavelength scale of each spectra was shifted to the rest frame of the galaxy.

The guided nuclear exposures were scaled by the effective exposure times on the nucleus during the drift scanning. These scaled exposures were then subtracted from the 
drift spectra. In this way, we separated the disk and nuclear contributions. Finally, all spectra were scaled to their median flux level.

\subsection{Errors}

The error calculation for $\left\langle\tilde{A}_{1}\right\rangle$ is described in RR98; it accounts for both photon statistics and systematic flat-fielding uncertainties.

Using Poisson statistics to describe the error in a raw spectrum we created an "error spectrum" and performed on it all of the standard reduction steps discussed in $§ 2.3 .2$ except for the cosmic ray cleaning. After normalizing the "science spectra," the "error spectra" were scaled by the median of the "science spectra" to form a detailed representation of the error at each pixel. The "error spectra" were used in all of the following analysis steps.

\section{SPECTRAL ANALYSIS \& STAR FORMATION HISTORY DIAGNOSTICS}

By examining spatially integrated spectral characteristics as a function of $\left\langle\tilde{A}_{1}\right\rangle$, we can determine whether lopsidedness affects the SF histories of galaxies. It is difficult, even in major mergers, to invert optical spectrophotometry into a SFH estimate (e.g. Turner 1998). However, the relative strength of the current and recent SFR in different galaxies may be studied with moderate $S / N$, non flux-calibrated spectra (e.g. Couch \& Sharples 1987; Barger et al. 1996). The equivalent width of $H \alpha$ in emission $\left(E W\left(H \alpha_{e m}\right)\right)$ integrated over the whole disk is a robust measure of the current global SFR in terms of the previously formed stars (Kennicutt et al. 1994). Much of the Balmer emission inn a galactic disk comes from the HII regions seen in SFR regions. In this regime, the Balmer decrement relates the emission flux in $H \beta$ to that in $H \alpha$. If the continuum level at these two wavelengths is similar, then the decrement will also relate the $E W s$ of the two lines. Therefore, the absorption corrected $E W\left(H \beta_{e m}\right)$ (see $\S 3.2$ ) serves as an indirect indicator of the current SFR in the disk.

The likely lifetime of lopsidedness has been estimated as $t_{\text {lop }} \approx 1 \mathrm{Gyr}$ (Baldwin et al. 1980; RZ95; ZR97). To examine SF on these timescales, we need a SF tracer with a comparable lifetime. Main sequence A-stars have lifetimes of $\approx 0.5$ Gyr (Clayton 1983), have strong spectral signatures (e.g. strong Balmer lines, a blue continuum, and a weak $4000 \AA$ break), and so serve as appropriate probes of the recent SFH. 


\section{1. $\quad$ Fitting Stellar Templates}

Fitting population synthesis models (e.g. Bruzual \& Charlot 1993) to our spectra can give us a measure of the recent SFH. However for four reasons we choose to simply fit with empirical stellar templates (Jacoby et al. 1984): 1) The spectral resolution of available population synthesis models $(\approx 10 \AA)$ is significantly less than that of our spectra $(3 \AA)$. 2) The empirical templates of Jacoby et al. (1984) have a spectral resolution $(4.5 \AA)$ slightly less than that of our spectra. 3) An adequate fit to the spectra can be obtained with a small number of high $\mathrm{S} / \mathrm{N}$ stellar templates. To estimate the recent, relative $\mathrm{SFH}$, we therefore need not deal with the complexities of the IMF and metallicity of the stellar populations.

As a qualitative measure of the relative contributions to our spectra from young and old stars, we synthesize the global absorption spectra of the galaxies in our sample with a linear combination of two stellar templates, an A0V and a G0III spectra from the Jacoby et al. (1984) stellar library. We also individually fit and subtract a $3^{\text {rd }}$ order polynomial of zero mean from the spectra and from each stellar template to correct for color terms (e.g. from calibration errors, from approximating the spectra with only two stellar templates). The spectra (with the polynomial subtracted) are fit by $\chi^{2}$ minimization with the following model:

$$
I_{\text {model }}(\lambda)=\left(C_{A 0 V} I_{A 0 V}(\lambda)\right) \otimes G(\sigma)+\left(C_{G 0 I I I} I_{G 0 I I I}(\lambda)\right) \otimes G(\sigma)-I_{\text {poly }}
$$

where $G(\sigma)$ and the weights, $\mathrm{C}$ were determined iteratively. $C_{A 0 V}$ and $C_{G 0 I I I}$ are the

weights for the normalized stellar template spectra, $I_{A 0 \mathrm{~V}}$ and $I_{G 0 I I I}$. $I_{\text {poly }}$ is the sum of the weighted template polynomial components, $G(\sigma)$ represents the Doppler broadening of the stars which is convolved with the stellar templates, and $\otimes$ is the convolution operator. For more details on the fitting procedure see Rix et al. (1995) and Turner (1998).

Independent of continuum slope, there is a unique set of line shapes and strengths for stars of each spectral type. The polynomial fit minimizes the effects of a global continuum slope on our best fit solution so that we are instead performing a global fit to the spectral features (4000 $\AA$ break, Ca H+K, Balmer series, etc.) We find that with only two, polynomial subtracted, stellar templates, we are able to consistently achieve fits with $\chi_{\nu}^{2} \leq 2$

\subsection{Emission Lines}

Our template fit to the galaxy spectra should reflect only the stellar populations in the galaxy, not interstellar emission. Therefore, we first fit all portions of spectra, omitting 
expected emission regions, and use the residual of the best model fit to construct an emission spectrum. We remove the large scale variations in the residual by fitting it with a high order polynomial $(\approx 50)$ at all locations where no emission is expected. We then fit each emission feature with a Gaussian. To isolate the stellar continuum, we subtract these gaussian components from the original spectra. We then re-fit our $I_{\text {model }}(\lambda)$ to this cleaned, pure absorption spectrum to determine $I_{\text {model }}^{\text {best }}(\lambda)$. This process is illustrated in Figure 1.

We measure $E W\left(H \beta_{\text {em }}\right.$ ) (see Table 2) from the complementary absorption corrected emission line spectra for all of our galaxies. A linear continuum was fit on either side of $H \beta\left(4720 \AA \leq \lambda_{B} \leq 4800 \AA ; 4900 \AA \leq \lambda_{R} \leq 4940\right)$. We then measured the equivalent width of the emission line integrating from $4843 \AA \leq \lambda_{\text {line }} \leq 4883 \AA$. The boundaries of our integration were chosen by visual inspection to minimize noise contributions from the continuum while maximizing the amount of line flux.

\subsection{Quantifying the A-star Fraction}

We can quantify the relative A star abundances by simply using the value of $C_{A 0 V}$ (see Table 2) in $I_{\text {model }}^{\text {best }}(\lambda)$. Because the basis templates are normalized to their median fluxes (as are the data,) the scaling factor of the individual templates gives a measure of how much A-stars contribute to the integrated spectra.

Balmer absorption lines are strongest in A-stars, the $4000 \AA$ break is weak, and so we may also use these two features to measure the recent SFH (Couch \& Sharples 1987; Barger et al. 1996). We use the equivalent width of $H \delta$ in absorption $\left(E W\left(H \delta_{a b s}\right)\right)$ as our indicator of Balmer line strength in order to minimize emission contamination and sample a relatively isolated region of the spectrum. A linear continuum was fit on either side of $H \delta\left(4000 \AA \leq \lambda_{B} \leq 4050 \AA ; 4150 \AA \leq \lambda_{R} \leq 4250 \AA\right)$. We measure the $E W$ itself from $4080 \AA \leq \lambda_{\text {line }} \leq 4120 \AA$.

Instead of measuring $E W\left(H \delta_{a b s}\right)$ directly from the spectrum, we decide to measure $E W_{\text {mod }}\left(H \delta_{a b s}\right)$ (see Table 2 ) of the best matching template spectrum, $I_{\text {model }}^{\text {best }}(\lambda)$. These template spectra are in general a good match to the data and provide an essentially noiseless estimate of $E W\left(H \delta_{a b s}\right)$ with a value derived from the best fit to a broad wavelength range. When measuring $E W\left(H \delta_{a b s}\right)$ directly from the spectra, the relation between $E W\left(H \delta_{a b s}\right)$ and $\left\langle\tilde{A}_{1}\right\rangle$ (see $\left.\S 4.3\right)$ clearly remains, but the scatter in $E W\left(H \delta_{a b s}\right)$ at a given $\left\langle\tilde{A}_{1}\right\rangle$ is slightly larger.

To further parameterize the recent SFH, we use the strength of the $4000 \AA$ break 
$\left(D_{4000}\right)$ (see Table 2$)$. Our measure of the break strength is defined as:

$$
D_{4000}=\frac{\int_{4050}^{4250} f_{\lambda} d \lambda}{\int_{3750}^{3950} f_{\lambda} d \lambda} .
$$

The suppression of the continuum blueward of $4000 \AA$ manifested in the break is caused by the combined absorption from the $\mathrm{Ca} \mathrm{H}+\mathrm{K}$ and $H \epsilon$ absorption line. As young, massive stars (with blue continua and weak metal lines) contribute more to the overall spectrum, $D_{4000}$ decreases.

\section{RESULTS AND DISCUSSION}

\subsection{Overall Spectral Characteristics}

To examine qualitatively the link between lopsidedness and SFR, we constructed composite disk spectra from the median of our nine most symmetric and nine most lopsided galaxies, and these we show in Figure 2. The lopsided composite spectrum has a much bluer continuum, stronger Balmer lines, stronger emission and a shallower $4000 \AA$ break than its symmetric counterpart regardless of exactly how many galaxies we include in the median spectrum. This result indicates that lopsidedness is in fact correlated with the recent SFH. However, a quantitative treatment of the relation is still needed.

\subsection{Is the Current Star Formation Correlated with Lopsidedness?}

Figure 3 shows the relation between $E W\left(H \beta_{e m}\right)$ and $\left\langle\tilde{A}_{1}\right\rangle$ for the entire spectroscopic sample. Using a Spearman-rank test, we find that the distribution of $E W\left(H \beta_{\text {em }}\right)$ vs. $\left\langle\tilde{A}_{1}\right\rangle$ deviates from a random one at the $99.9 \%$ level. The median $E W\left(H \beta_{e m}\right)$ of our nine most symmetric galaxies is $-3.2 \AA$ while the median for our nine most lopsided galaxies is $-6.3 \AA$. The correlation of $E W\left(H \beta_{e m}\right)$, and hence the current SFR, with $\left\langle\tilde{A}_{1}\right\rangle$ is clearly shown in Fig. 3. This implies that an elevation in the instantaneous SFR occurs over the same timescales as lopsidedness, lasting maybe as long as 1 Gyr after the onset of lopsidedness.

\subsection{Is Recent Star Formation Correlated with Lopsidedness?}

Figure 4 shows the relation of A-star content, $E W_{\text {mod }}\left(H \delta_{a b s}\right)$, and $D_{4000}$ with $\left\langle\tilde{A}_{1}\right\rangle$. They deviate from random distributions at the $99.997 \%, 99.99 \%$, and $99.68 \%$ levels respectively. 
Our sample is not large enough to study these correlations separately for different Hubble types; instead we analyze the change in spectral properties of the sample as a whole. Figure 5 shows that the data are differentiated by lopsidedness in the $E W_{\text {mod }}\left(H \delta_{a b s}\right)$ vs. $D_{4000}$ plane. The large scatter is not surprising, as even within a single Hubble type there is a considerable variation in the specific SFHs (Kennicutt et al. 1994). Between the most lopsided and symmetric $1 / 3$ of our galaxies the median values of $E W_{\bmod }\left(H \delta_{a b s}\right)$ and $D_{4000}$ differ by, $\Delta E W_{\text {mod }}\left(H \delta_{a b s}\right)=2.1 \pm 1.0 \AA$ and $\Delta D_{4000}=0.24 \pm 0.01$, respectively. This defines a boost vector (Fig. 5) whose direction and magnitude characterize the difference in spectral indices between a symmetric and lopsided galaxy in our sample.

\subsection{Constraining the Boost Mass}

A number of efforts have shown that the detailed SFH, even over the last $10^{9}$ years, cannot be determined unambiguously from integrated spectra (e.g. Turner 1998 and references therein; Leonardi \& Rose 1996). Instead, we restrict ourselves to a set of model SFHs consisting of a "normal" or "underlying" spiral galaxy SFH, which in lopsided galaxies has been boosted in the past by a factor $C_{\text {boost }}$. We then attempt to characterize the boost strength,$C_{\text {boost }}$, and an associated timescale. Using the EPS code of Bruzual \& Charlot, (GISSEL 1995; in preparation) we construct SEDs representing the light from the underlying stellar populations of galaxies in our sample. We specify the SFH by the birthrate parameter $b$ (Scalo 1986), where

$$
b=\frac{\mathrm{SFR}_{\text {current }}}{\left\langle\mathrm{SFR}_{\text {past }}\right\rangle} .
$$

Assuming an exponential SFH, i.e. $S F R(t) \propto \exp [-t / \tau(b)]$, each $b$ has an associated timescale, $\tau(b)$ defined through

$$
\exp \left[\frac{t_{g a l}}{\tau(b)}\right]-1=\frac{t_{g a l}}{\tau(b)} \frac{1}{b},
$$

with $t_{\text {gal }}$ as the age of the galaxy. We adopt an age of $t_{\text {gal }} \approx 10 \mathrm{Gyr}$ and a metallicity of $Z=Z_{\odot}$. We also use the Salpeter $(1955) \mathrm{IMF}$ with a mass range of $0.1 M_{\odot}-125 M_{\odot}$. This IMF provides a better fit than does the Scalo IMF (1986) to the global photoionization rates and to the colors of galactic disks (Kennicutt et al. 1994). Applying the Scalo IMF results in slightly lower fractional boost masses than the Salpeter IMF, but the IMF choice does not qualitatively affect our conclusions. The model SED is then given by the integral:

$$
F(\tau(b), \lambda)=\int_{0}^{t_{g a l}} S\left(t^{\prime}, \lambda\right) r\left(\tau(b), t^{\prime}\right) d t^{\prime}
$$


where

$$
r(\tau(b), t)=\beta(b) \exp \left[-\frac{t}{\tau(b)}\right],
$$

where $\beta(b)$ is the Hubble type dependent initial SFR of the galaxy used to normalize the present day masses to the same value, and where $S\left(t^{\prime}, \lambda\right)$ is the SED for a population $t^{\prime}$ years after a delta function burst (Bruzual \& Charlot's simple starburst model).

We account for the change in the spectral properties by adding an exponentially declining increase ,or "boost" in SF on top of the underlying galactic SFH. We assume that the boost originated about $5 \times 10^{8}$ years ago, comparable to the presumed dynamical age of the boost and the A-stars seen in Figure 2. The model SED for a galaxy with a boost population is given by:

$$
F(\tau(b), \lambda)=\int_{0}^{t_{g a l}} S\left(t^{\prime}, \lambda\right) \beta(b) \exp \left[-\frac{t^{\prime}}{\tau(b)}\right] d t^{\prime}+\int_{0}^{t_{\text {boost }}} S\left(t^{\prime}, \lambda\right) C_{\text {boost }} \exp \left[-\frac{t^{\prime}}{\tau_{\text {boost }}}\right] d t^{\prime}
$$

For each Hubble type, i.e. for each $\tau(b)$ we construct model tracks by varying $C_{\text {boost }}$ and $\tau_{\text {boost }}$ in Equation (9), effectively adding differing boosts to the unperturbed galaxy SFH. As shown in Fig. 6, moving the underlying population later in Hubble type, or to a larger $\tau(b)$, shifts the tracks to greater values of $E W_{\text {mod }}\left(H \delta_{a b s}\right)$ and smaller values of $D_{4000}$. For a given Hubble type, varying the boost timescale changes the slope of the track of increasing boost mass.

It is interesting to note that our empirical boost vector cannot be matched directly with any of the models in the $E W_{\text {mod }}\left(H \delta_{a b s}\right)$ vs. $D_{4000}$ plane, suggesting that the EPS models may have systematic errors. Assuming, however, that the correct models will differ from these by a simple shift in the $E W_{\text {mod }}\left(H \delta_{a b s}\right)$ vs. $D_{4000}$ plane, we choose the underlying population model which minimizes the distance (in the $E W_{\text {mod }}\left(H \delta_{a b s}\right)$ vs. $D_{4000}$ plane) to the beginning of our observed boost vector. We then match the slope of the boost vector by varying the boost timescale. The best match we find was for $b=0.33$ (Hubble type Sb; Kennicutt et al. 1994) and $\tau_{\text {boost }}=500 \mathrm{Myr}$. For reference, we also show the tracks for underlying galaxy spectra with $b$ parameters corresponding to Hubble types of Sab and Sbc ( $b=0.17$ and 0.84 respectively; Kennicutt et al. 1994). For each of these Hubble types, we plot tracks corresponding to boosts with $\tau_{\text {boost }}=100 \mathrm{Myr}$ and $\tau_{\text {boost }}=500 \mathrm{Myr}$. We also plot the $\tau_{\text {boost }}=100$ Myr boost for the $b=0.33$ model. Measuring the projected length of the boost vector on the best model, and assuming the galaxy disk has a final stellar mass of $10^{10} M_{\odot}$, we find that $\sim 1 \times 10^{9} M_{\odot}$ of stars have been formed over the the past $0.5 \mathrm{Gyr}$ in addition to the "underlying" SFH. This corresponds to an factor of $\sim 8$ increase in the SFR over the past $5 \times 10^{8}$ years. 
Extinction will have two primary effects on our data. It will redden the continuum from the galaxy. By allowing for a polynomial element to our template fits, we minimize the effect of the continuum slope on our results. Extinction may also affect regions of current $\mathrm{SF}$; obscuring both emission lines and the continuum from massive young stars. We do not make a further account of reddening but acknowledge that our boost masses may increase if reddening is included.

\subsection{Correlations in Disguise}

It is important to verify that our result is not an artifact of correlations between the underlying SFH and lopsidedness. To determine whether mass, gas content or Hubble type effects mimic our relation between lopsidedness and SFR, we examined the distributions of $E W\left(H \beta_{e m}\right), E W_{\text {mod }}\left(H \delta_{a b s}\right), D_{4000}$, A star content and $\left\langle\tilde{A}_{1}\right\rangle$ vs. $L_{B}, M_{H I} / L_{B}$ and T-type (De Vaucouleurs et al. 1991). A hidden correlation exists if any of the latter three parameters are correlated both with a SF indicator and with $\left\langle\tilde{A}_{1}\right\rangle$.

None of the above distributions, except for $E W\left(H \beta_{e m}\right)$ and $D_{4000}$ vs. T-type and $M_{H I} / L_{B}$, have more than a $1.7 \sigma$ probability of being correlated, with a $\leq 2.6 \sigma$ significance for the four remaining correlations. Given the known correspondence between SFR and Hubble type, mean stellar population and Hubble type, and SFR and gas surface density (Kennicutt et al. 1994), these correlations with $E W\left(H \beta_{e m}\right)$ and $D_{4000}$ are expected. However, none of our canonical galaxy parameters show a correlation with lopsidedness at above the $1.2 \sigma$ level and we can therefore be confident that we are seeing a true correlation between our SF indicators and $\left\langle\tilde{A}_{1}\right\rangle$.

\subsection{Nuclear Properties and Contributions}

As an initial diagnostic of the nuclear contributions, we examine the fraction of galactic light originating in the nucleus. Even without absolute spectrophotometry, we can still examine what percentage of the total galaxy light is made up of light from the nucleus as long as the spectra are calibrated with respect to each other.

The fractional luminosity of the nucleus ranged from $0.1-6.4 \%$ with a median value of $1.7 \%$. The fractional luminosity of the nucleus is uncorrelated with $\left\langle\tilde{A}_{1}\right\rangle$. A Spearman-Rank test showed that there was a $52 \%$ probability that the data was drawn from a random sample. Figure 7 shows that while the nuclear $E W\left(H \beta_{\text {em }}\right)$ vs. $\left\langle\tilde{A}_{1}\right\rangle$, and $E W_{\text {mod }}\left(H \delta_{a b s}\right)$ vs. $\left\langle\tilde{A}_{1}\right\rangle$ are correlated, the relations show more scatter than in the disk. The Spearman-Rank 
test shows that these two relations are less significantly correlated in the nucleus than in the disk by $0.4 \sigma$ and $0.7 \sigma$, respectively. Only for $D_{4000}$ vs. $\left\langle\tilde{A}_{1}\right\rangle$ was the correlation greater than in the disk.

Our data indicate that the enhanced SF in lopsided galaxies occurs in the disk with equal or greater strength than in the nucleus. This result appears to run contrary to what numerical simulations suggest about the reactions of gas in the disk to tidal perturbations from external sources. Mihos \& Hernquist (1994) and Hernquist \& Mihos (1995) show that even high mass ratio mergers $\left(M_{\text {gal }} / M_{\text {sat }}>10\right)$ can form a bar which torques gas in the disk and funnels much of it into the nucleus. However the presence of a dense bulge easily inhibits bar formation and hence gas is not funneled as effectively. Another explanation is that star formation is occurring in large amounts at the nucleus, but is partly masked by extinction. High extinction is often found to be coincident with high star formation regions in mergers and is easily capable of hiding the presence of young populations (Turner 1998).

\subsection{The Frequency of Lopsidedness- Revisited}

To estimate the volume density of lopsided galaxies from a magnitude limited sample requires the understanding of any systematic differences in luminosity between lopsided and symmetric galaxies (ZR97). Our best fit boost brightens the galaxies by 1.3, 1.0 and 0.8

magnitudes (in B, V, and R, respectively) over the brightness of the underlying population. Lopsided galaxies will hence be overrepresented in our B-band selected sample, and their volume density will be $\sim 25 \%$ of that derived from any magnitude limited survey. To compare the frequency of lopsidedness for different Hubble types (e.g. RR98), we need to understand how this brightening depends on the "underlying" SFH. Unfortunately, we have too few galaxies in our sample to discuss Hubble type dependent effects on the boost strength and luminosity evolution.

At present, the main constraint on the frequency of lopsidedness is from its frequency in imaging samples (20\%; RR98). Coupled with our brightening estimates, we find that $\sim 5 \%$ of the galaxies in a volume limited sample will be lopsided. Lopsidedness is likely caused by minor mergers, the frequency of which increases with look back time. Given that lopsidedness lasts for $\sim 1$ Gyr, we can estimate that the average galaxy has been lopsided at least once in its lifetime. 


\section{SUMMARY \& CONCLUSION}

To quantify the correlation between the recent SF histories of present-day spiral galaxies and their global asymmetry, we compare the integrated spectral properties of late-type spirals of varying lopsidedness. We find that the recent $(\leq 0.5 \mathrm{Gyr}) \mathrm{SFH}$ and current $\left(\leq 10^{7}\right.$ years) SFR are both strongly correlated with $\left\langle\tilde{A}_{1}\right\rangle$ although there is appreciable scatter in the individual galaxy-to-galaxy properties. For $E W\left(H \beta_{e m}\right)$, reflecting the current SFR, we find a $3.2 \sigma$ Spearman-rank correlation with $\left\langle\tilde{A}_{1}\right\rangle$. We fit a combination of A0V and G0III stellar spectra to our galaxy spectra to quantify the relative abundance of A-stars in the disk (which traces the SFR within 0.5 Gyrs). From these best fit model spectra, $I_{\text {model }}^{\text {best }}(\lambda)$, we measure a number of spectral indices, and find that $E W_{\bmod }\left(H \delta_{a b s}\right), D_{4000}$, and $C_{A 0 V}$ are correlated with $\left\langle\tilde{A}_{1}\right\rangle$ at the $3.9 \sigma, 3.0 \sigma$, and $4.2 \sigma$ levels, respectively.

We measure the same spectral indices in the nucleus, and find them less correlated with $\left\langle\tilde{A}_{1}\right\rangle$ (except $D_{4000}$ ). Unless a nuclear starburst is obscured, the disk and not the nucleus is the primary site of the SF increase we see in lopsided galaxies. This is in contrast to numerical simulations where minor mergers funnel gas into the nucleus of galaxies, causing intense starbursts (Mihos \& Hernquist 1994; Hernquist \& Mihos 1995). Only by the presence of a dense bulge can the formation of a bar, and the subsequent funneling of gas, be prevented.

To quantify the mass of additional stars formed in lopsided galaxies, we defined a boost vector in $E W_{\text {mod }}\left(H \delta_{a b s}\right)$ vs. $D_{4000}$ space, by comparing the median values of these properties for the most symmetric third and the most lopsided third of our sample. We find $\Delta E W_{\text {mod }}\left(H \delta_{a b s}\right)=2.1 \pm 1.0 \AA$ and $\Delta D_{4000}=0.024 \pm 0.01$. We fit this vector with an "underlying population + boost" EPS model corresponding to a progenitor galaxy with $b=0.33, \tau_{\text {boost }}=500 \mathrm{Myr}$, and boost age of 0.5 Gyr. Using this best fit EPS model, we find that $\sim 1 \times 10^{9} M_{\odot}$ is formed in the boost in addition to the "underlying" SFH (assuming a final stellar mass of $\left.10^{10} M_{\odot}\right)$. This is a considerable fraction $(\sim 10 \%)$ of the final stellar mass of the galaxy and corresponds to a factor of 8 increase in the SFR over the past $5 \times 10^{8}$ years. Given the increasing merger rates and increasing gas fractions towards higher redshifts, minor merger induced SF boosts of short duration played an important role in assembling the present day stellar content of galaxies.

Finally, we address by how much the frequency of lopsidedness from a magnitude limited sample is increased by the corresponding luminosity boost. Our best fit EPS boost model corresponds to a $\sim 1$ magnitude brightening when galaxies becomes lopsided, increasing their presence four-fold in magnitude limited samples. We lack the statistics however, to examine any Hubble type dependent differences in the luminosity boost. 
It is obvious that more work needs to be done to fully understand the cause of lopsidedness as well as the SFH of lopsided galaxies. To quantify the Hubble type specific boost in the recent SFH, a large sample should be obtained with significant numbers of galaxies in each Hubble type bin. Since imaging and spectroscopy will be needed for this project, a volume limited sample may be constructed which bypasses many of the problems encountered when selecting galaxies according to an apparent magnitude limit. Companion searches to sufficiently faint magnitudes will help to study the possible link between environment and lopsidedness (as caused by weak tidal interactions). With the recent commissioning of large area imaging and spectroscopy surveys such as Sloan Digital Sky Survey, constructing such a sample will become relatively straightforward.

Numerical simulations have shown to be a useful tool in studying the evolution of the stellar and gas distributions in minor mergers. High resolution simulations with a live halo are crucial for studying the detailed response of the disk to the merger (Walker et al. 1996). A thorough exploration of interaction parameter space is needed to quantify the structural and kinematic response in the stellar and gas components. High resolution N-body studies are also needed to explore the global stability of isolated galactic disks.

This work was completed with partial support from NSF grants AST9870151, AST9421145 and AST9900789. Greg Rudnick and H.-W. Rix would like to thank Nelson Caldwell for many valuable discussions on measuring SFHs from integrated spectra. Greg Rudnick would like to thank Craig Kulesa and Christopher Gottbrath for many useful discussions in the early hours of the morning. Greg Rudnick would also like to thank Megan Sosey and Chris Gottbrath for assisting with our observing program. Finally, we would like to thank the Steward Observatory 2.3-m telescope Operators for their assistance in the completion of this project. 


\section{REFERENCES}

Allam, S. S., Tucker, D. L., Lin, H., \& Hashimoto, Y. 1999, ApJ, 522, L89

Baldwin, J. E., Lynden-Bell, D., \& Sancisi, R. 1980, MNRAS, 193, 313

Barger, A. J., Aragon-Salamanca, A., Ellis, R. S., Couch, W. J., Smail, I., \& Sharples, R. M. 1996, MNRAS, 279, 1

Barnes, J., \& Hernquist, L. 1991, ApJ, 370, L65

Barnes, J., \& Hernquist, L. 1992, ARA\&A, 30, 705

Barnes, J., \& Hernquist, L. 1996, ApJ, 471, 115

Bruzual, A. G., \& Charlot, S. 1993, ApJ, 405, 538

Barton, E. J., Bromley B. C., \& Geller, M. J. 1999, ApJ, 511, L25

Clayton, D. 1983, Principles of Stellar Evolution and Nucleosynthesis. Chicago: Univ. of Chicago Press

Couch W. J., \& Sharples, R. M. 1987, MNRAS, 229, 423

De Vaucouleurs, G., De Vaucouleurs, A., Corwin, H. G., Jr., Buta, R. J., Paturel, G., \& Fouque, P. 1991, Third Reference Catalog of Bright Galaxies. New York: Springer-Verlag

Hashimoto, Y., Oemler, A., Lin, H., \& Tucker, D. L. 1998, ApJ, 499, 589

Haynes, M. P., van Zee, L., Hogg, D. E., Roberts, M. S., \& Maddalena, R. J. 1998, AJ, 115, 62

Hernquist, L., Heyl, J., \& Spergel, D. 1993, ApJ, 416, L9

Hernquist, L., \& Mihos, C. J. 1995, ApJ, 448, 41

Jacoby, G. H., Hunter, D. A., \& Christian, C. A. 1984, ApJS, 56, 257

Johnston, K. V., Hernquist, L., \& Bolte, M. 1996, ApJ, 465, 278

Kennicutt, R. C., Jr., Roettiger, K. A., Keel, W. C., Van Der Hulst, J. M., \& Hummel, E. 1987, AJ, 93, 1011

Kennicutt, R. C., Jr. 1992, ApJ, 388, 310 
Kennicutt, R. C., Jr., Tamblyn, P., \& Congdon C. W. 1994, ApJ, 435, 22

Kennicutt, R. C., Jr. 1998, ApJ, 498, 541

Lacey, C., \& Cole, S. 1993, MNRAS, 262, 627

Larson, R. B., \& Tinsley, B. M. 1978, ApJ, 219, 46

Leonardi, A. J., Rose, J. A. 1996, AJ, 111, 182L

Levine, S. E., \& Sparke, L. S. 1998, ApJ, 496, 13

Mihos, C. J., Richstone, D. O., \& Bothun, G. D. 1992, ApJ, 400, 153

Mihos, C. J., \& Hernquist, L. 1994, ApJ, 425, L13

Quinn, P. J., Hernquist, L., \& Fullagar, D. P. 1993, ApJ, 403, 74

Richter, O.-G., \& Sancisi, R. 1994 A \& A, 290, L9

Rix, H.-W., \& Zaritsky, D. 1995, ApJ, 447, 82

Rix, H.-W., Kennicutt, R. C., Jr., Braun, R., \& Walteros, R. A. M. 1995, ApJ, 438, 155

Roberts, M. S., \& Haynes, M. P. 1994, ARA\&A, 32, 115

Rudnick, G., \& Rix, H.-W. 1998, AJ, 116, 1163

Salpeter, E. E. 1955, ApJ, 121, 161

Sanders, D. B., Soifer, B. T., Elias, J. H., Madore, B. F., Matthews, K., Neugebauer, G., \& Scoville, N. Z. 1988, ApJ, 325, 74

Scalo, J. M. 1986, Fund. Cos. Phys., 11, 1

Searle, L., \& Zinn, R. 1978, ApJ, 225, 357

Sellwood, J. A., \& Merritt, D. 1994, ApJ, 425, 530

Swaters, R. A., Schoenmakers, R. H. M., Sancisi, R., \& van Albada, T. S. 1999, MNRAS, 304,330

Syer, D., \& Tremaine, S. 1996, MNRAS, 281, 925

Toomre, A., \& Toomre, J. 1972, ApJ, 178, 623

Toth, G., \& Ostriker, J. P. 1992, ApJ, 389, 5 
Turner, A. 1998, PhD. Thesis

Walker, I. R., Mihos, C. J., \& Hernquist, L. 1996, ApJ, 460, 121

Weinberg, M. D. 1994, ApJ, 421, 481

Zang, T. A., \& Hohl, F. 1978, ApJ, 226, 521

Zaritsky, D., \& Rix, H.-W. 1997, ApJ, 477, 118

This preprint was prepared with the AAS IATEX macros v4.0. 


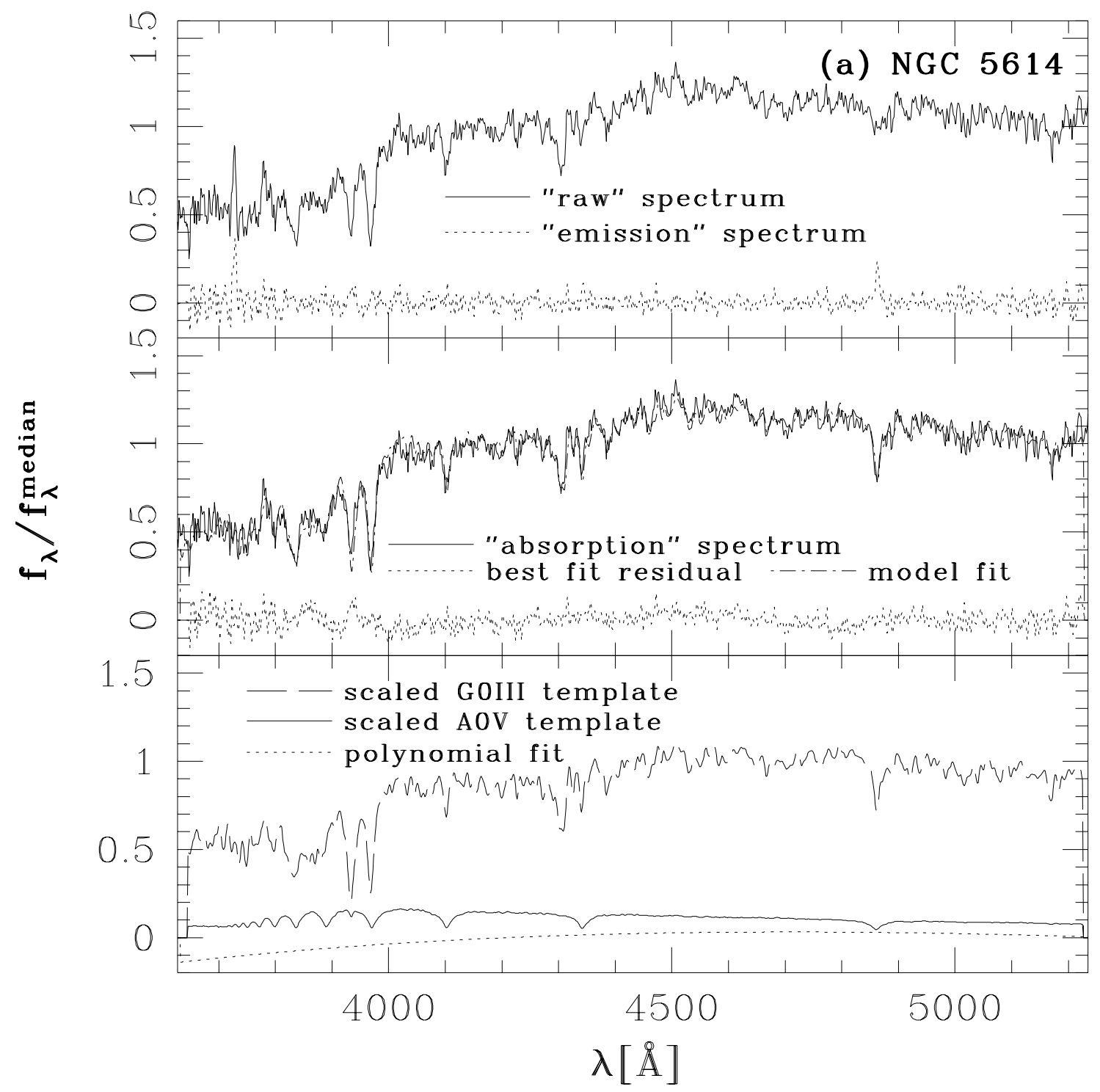

Fig. 1.- Empirical model fits (G0III + A0V) for two sample galaxies: (a) NGC 5614, in which the older population (modeled by a G0III star) clearly dominates the spectrum and (b) NGC 4595, whose stellar light is made up in large part, of light from young massive stars (modeled by a A0V star). The top panel shows the "disk" spectrum prior to emission line decomposition. It also shows the "emission" spectrum created from the best fit residual of the first pass through the fitting routine. The middle panel shows the "absorption" spectrum, the best fit model, $I_{\text {model }}^{\text {best }}(\lambda)$, and the resulting fit residual. The bottom panel gives the weighted, doppler broadened stellar templates and the total 3rd order polynomial contribution whose sum creates the model seen in the middle panel. 


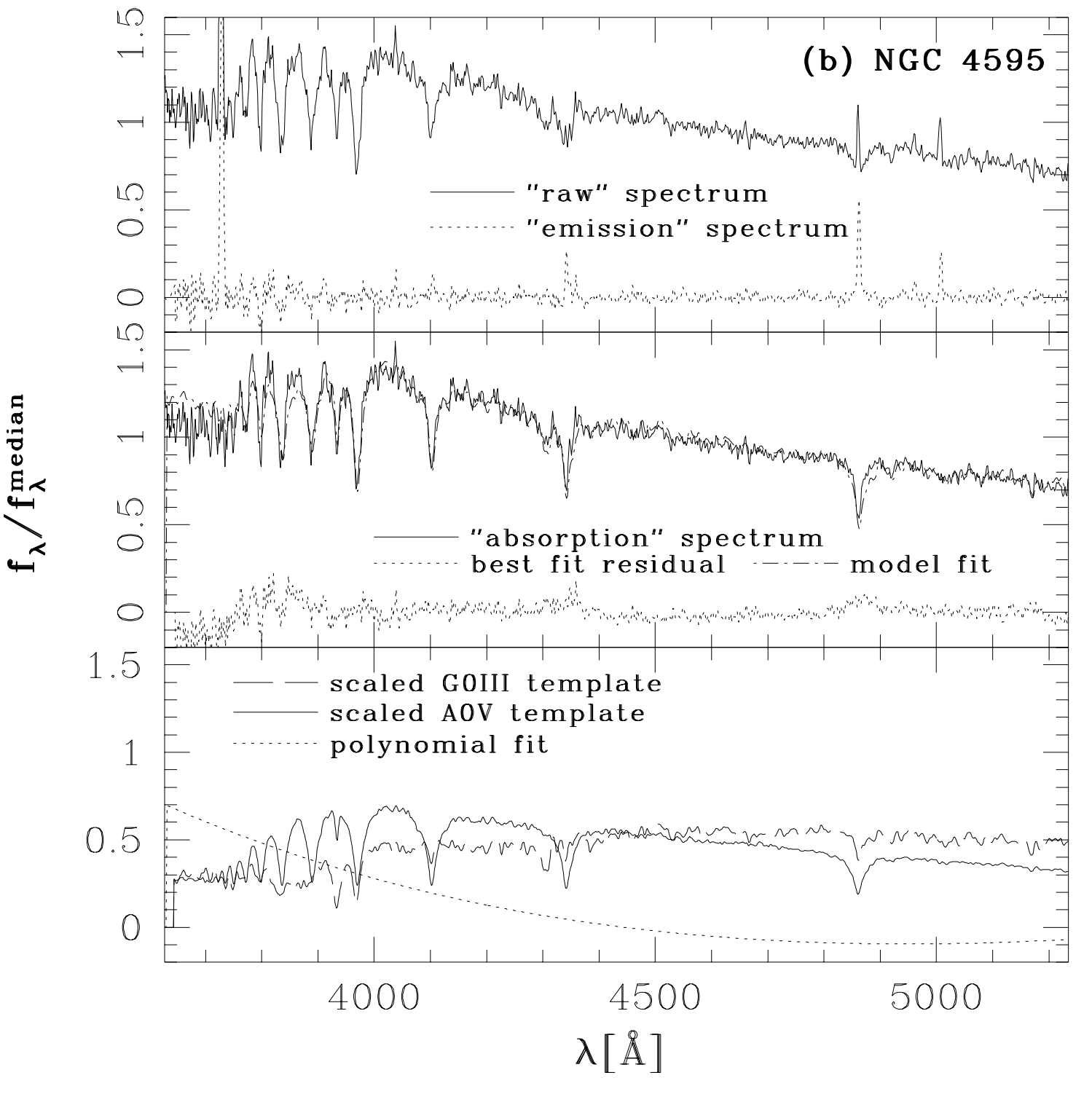




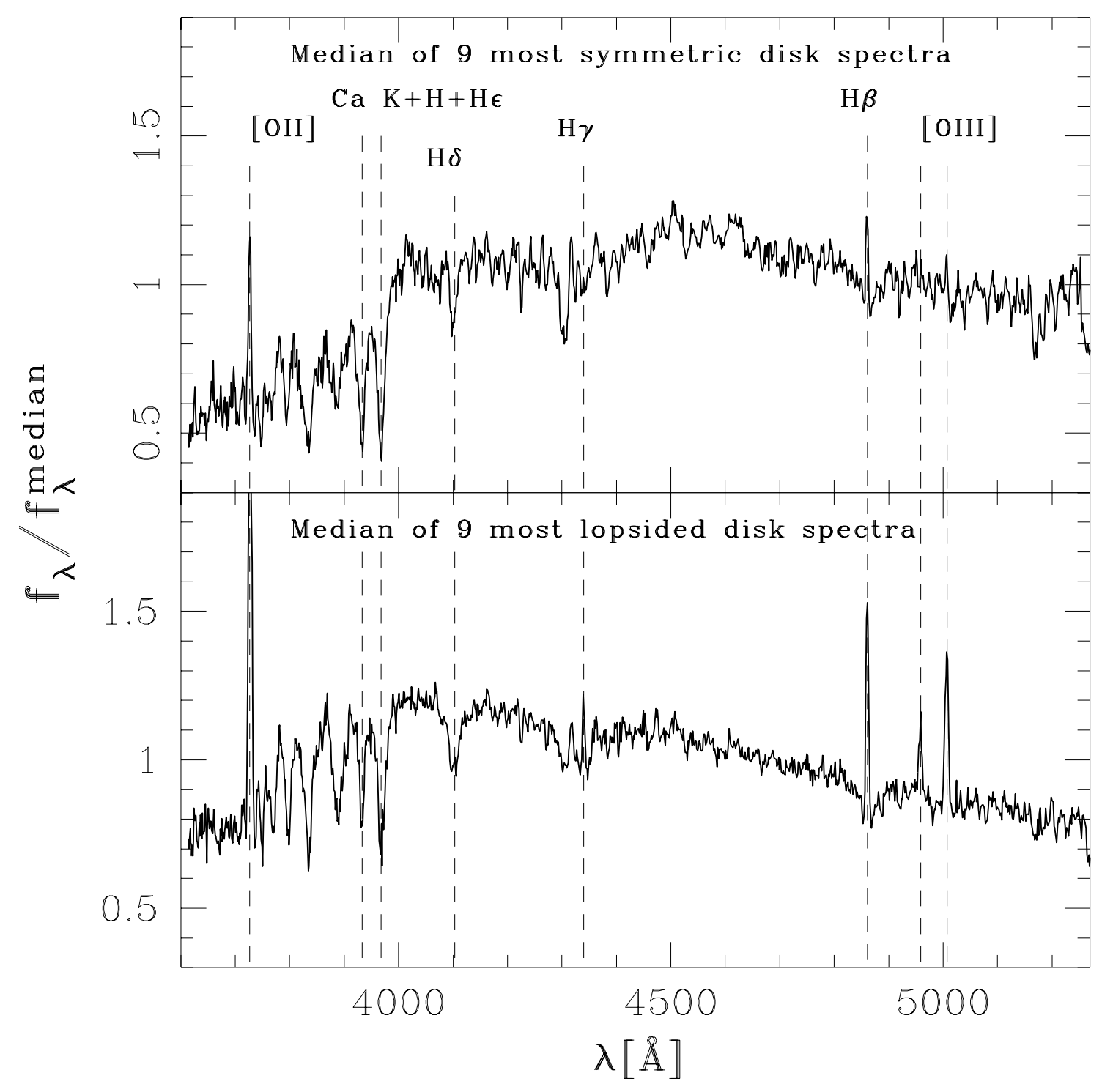

Fig. 2.- The composite, median spectrum of our nine most symmetric (top) and nine most lopsided (bottom) galaxies. Note that the lopsided composite has a bluer continuum, stronger Balmer absorption lines, stronger emission lines and a shallower $4000 \AA$ break than the symmetric composite. The median values of $\left\langle\tilde{A}_{1}\right\rangle$ for the symmetric and lopsided composites are 0.04 and 0.33 respectively. 


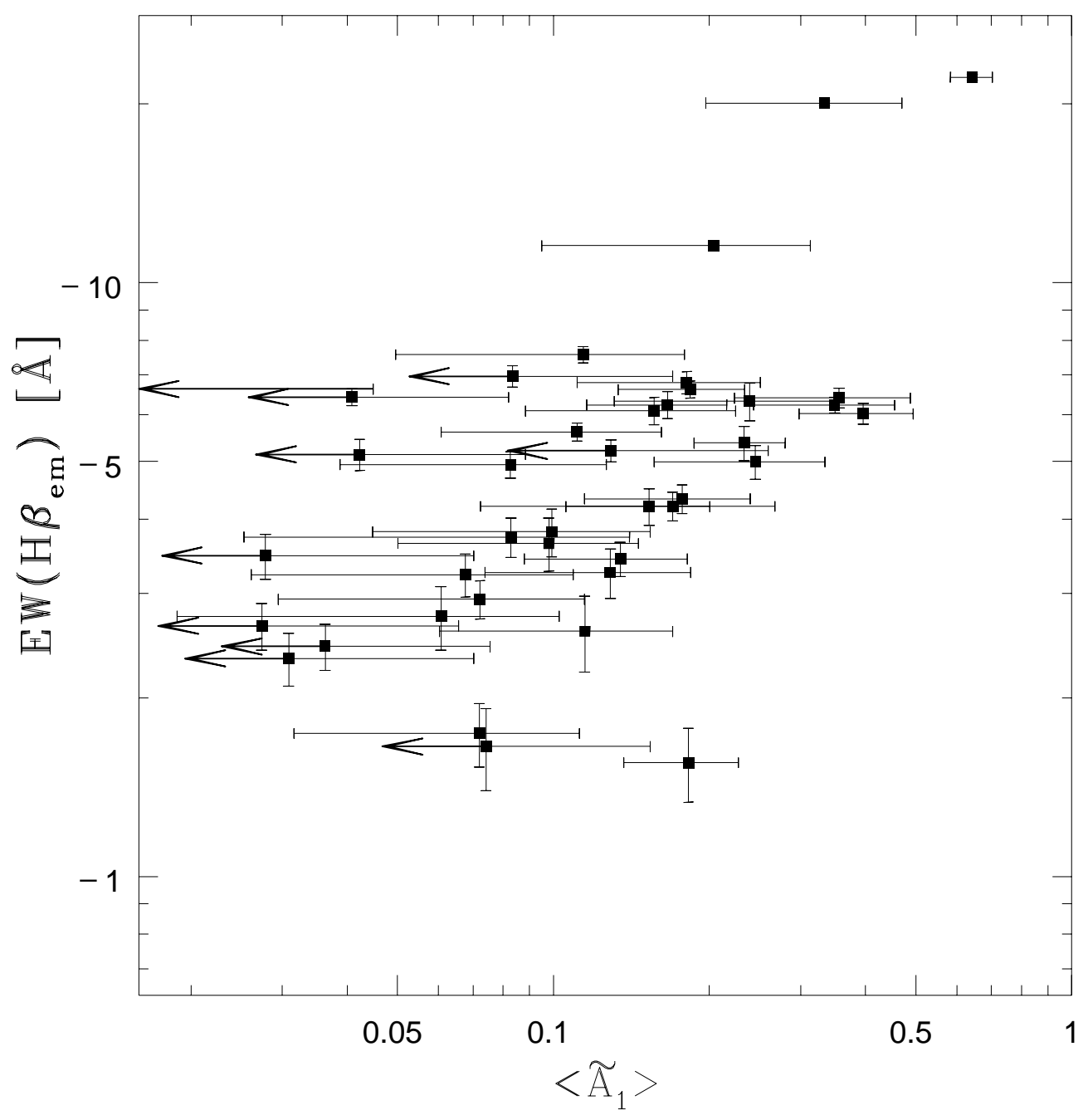

Fig. 3.- The $E W\left(H \beta_{e m}\right)$, integrated over the disks, as measured from the decomposed "emission" spectrum of each galaxy. Arrows on data points indicate that the errors are larger than the values themselves and that the values should be therefore be treated as upper limits. 


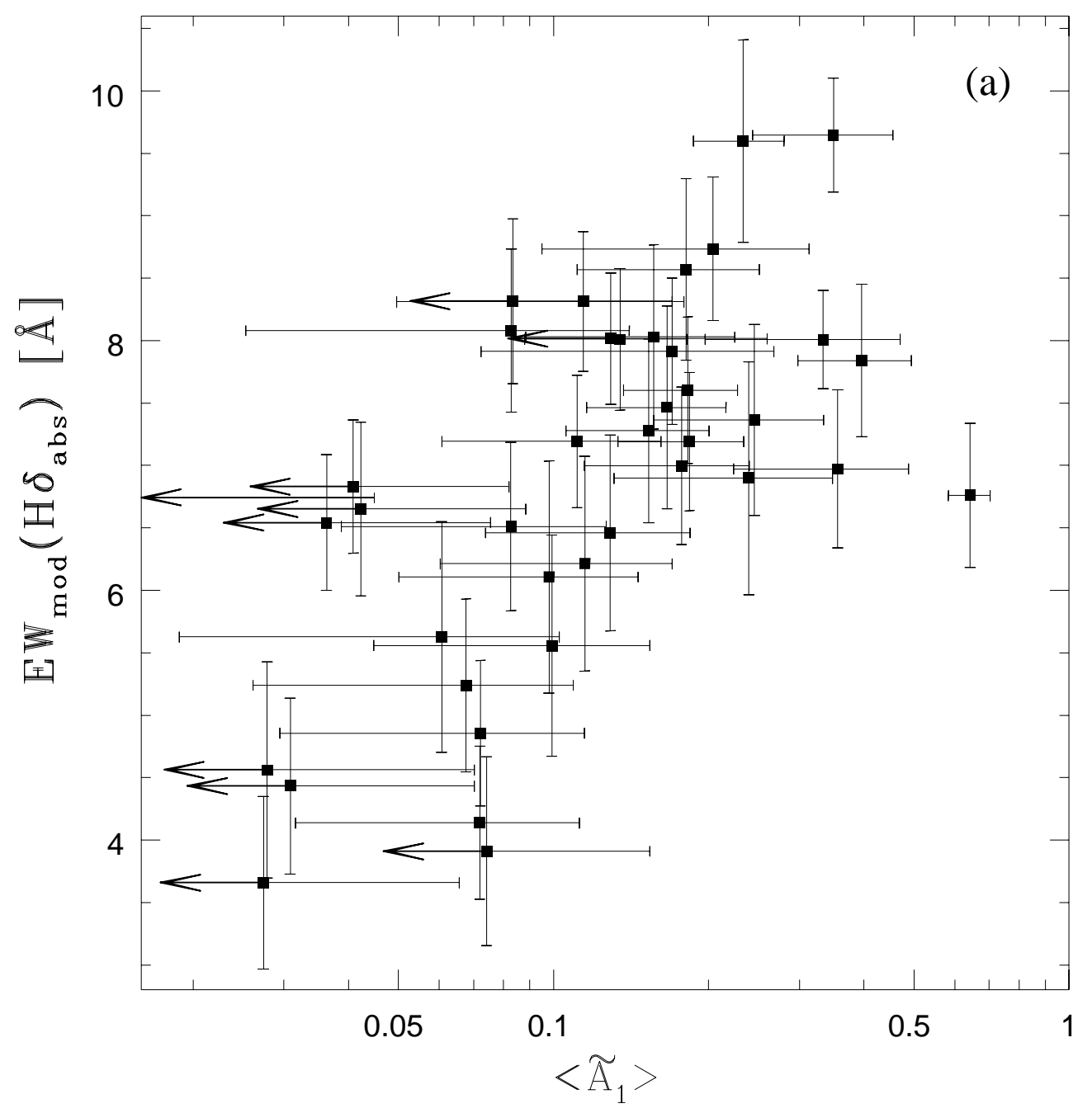

Fig. 4.- Measures of the recent SFH vs. $\left\langle\tilde{A}_{1}\right\rangle$ : (a) $E W_{m o d}\left(H \delta_{a b s}\right)$ as measured from our best fit stellar template model to the "absorption" spectrum of the galaxy. (b) The value of $D_{4000}$ as measured from our "absorption" spectra. (c) The scaling factor of the A star template $\left(C_{A 0 V}\right)$ in the best model fit to the "absorption" spectrum of the galaxy $I_{\text {model }}^{\text {best }}(\lambda)$. Arrows on data points indicate upper limits. 


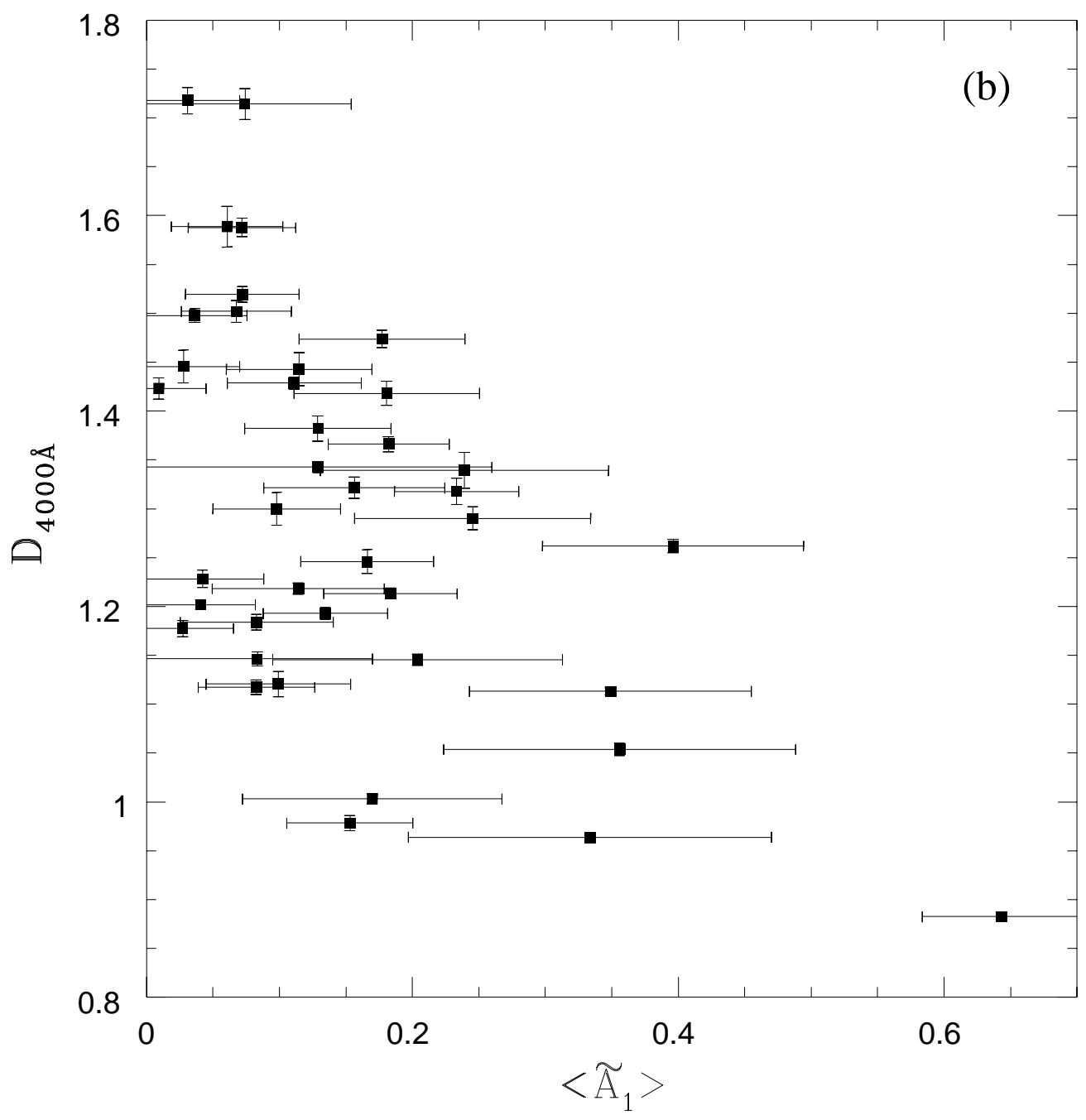




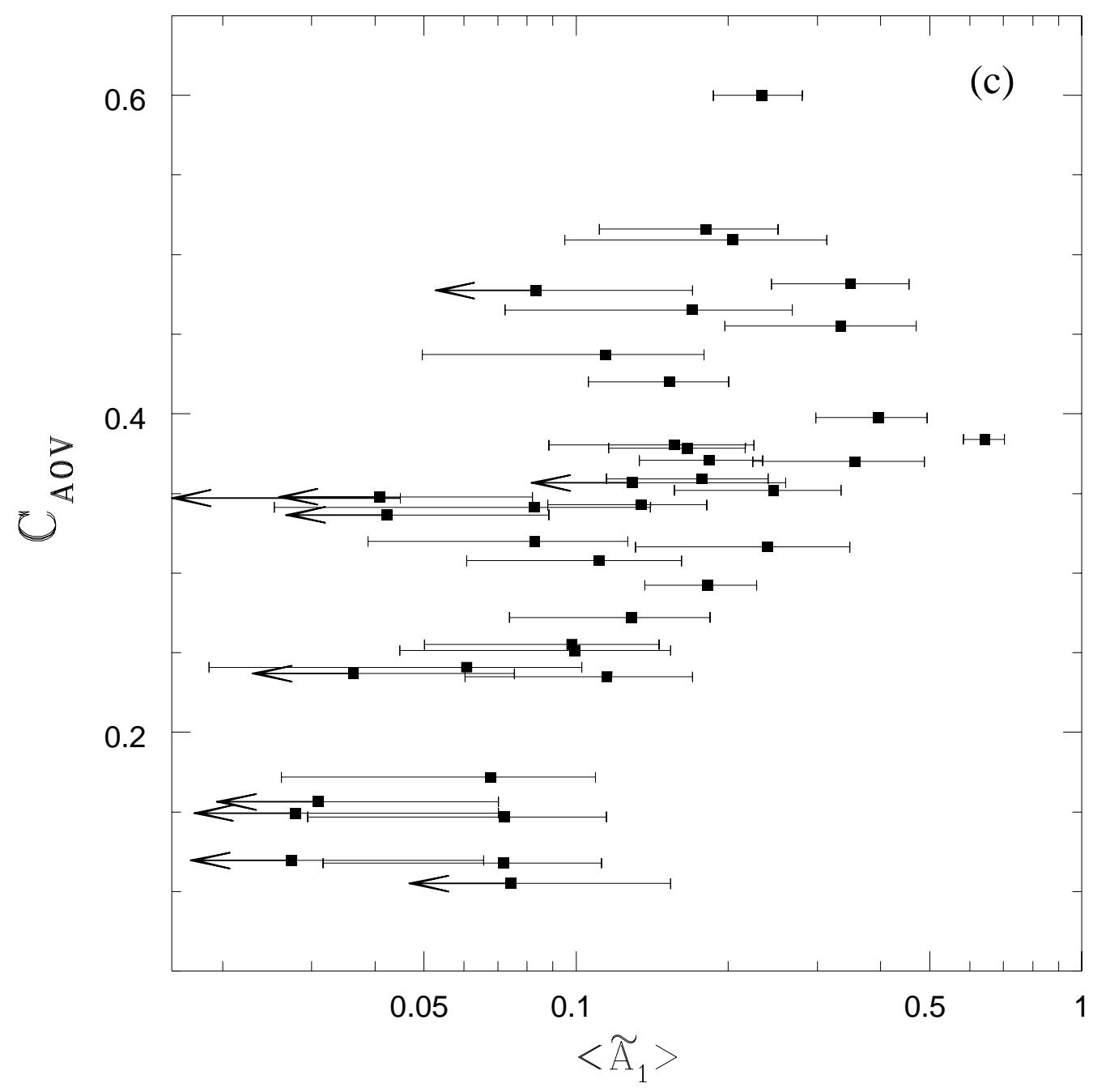




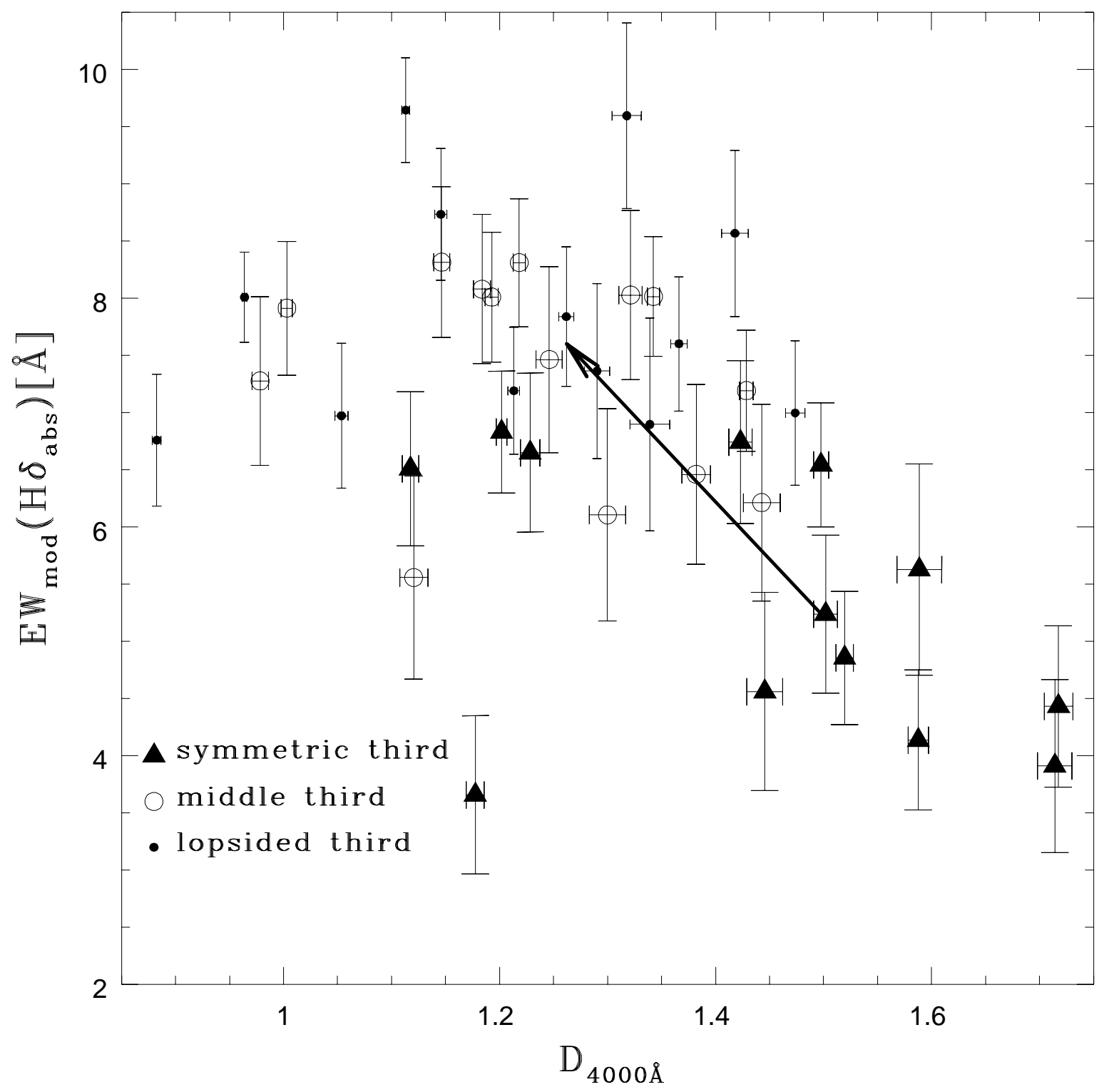

Fig. 5.- The values for all of the galaxies plotted in the $E W_{\text {mod }}\left(H \delta_{a b s}\right)$ vs. $D_{4000}$ plane. We split the data into the most, middle and least lopsided thirds of the sample. We also overlay the boost vector connecting the median values of the spectral indices for the most symmetric third, to the median values for the most lopsided third. 


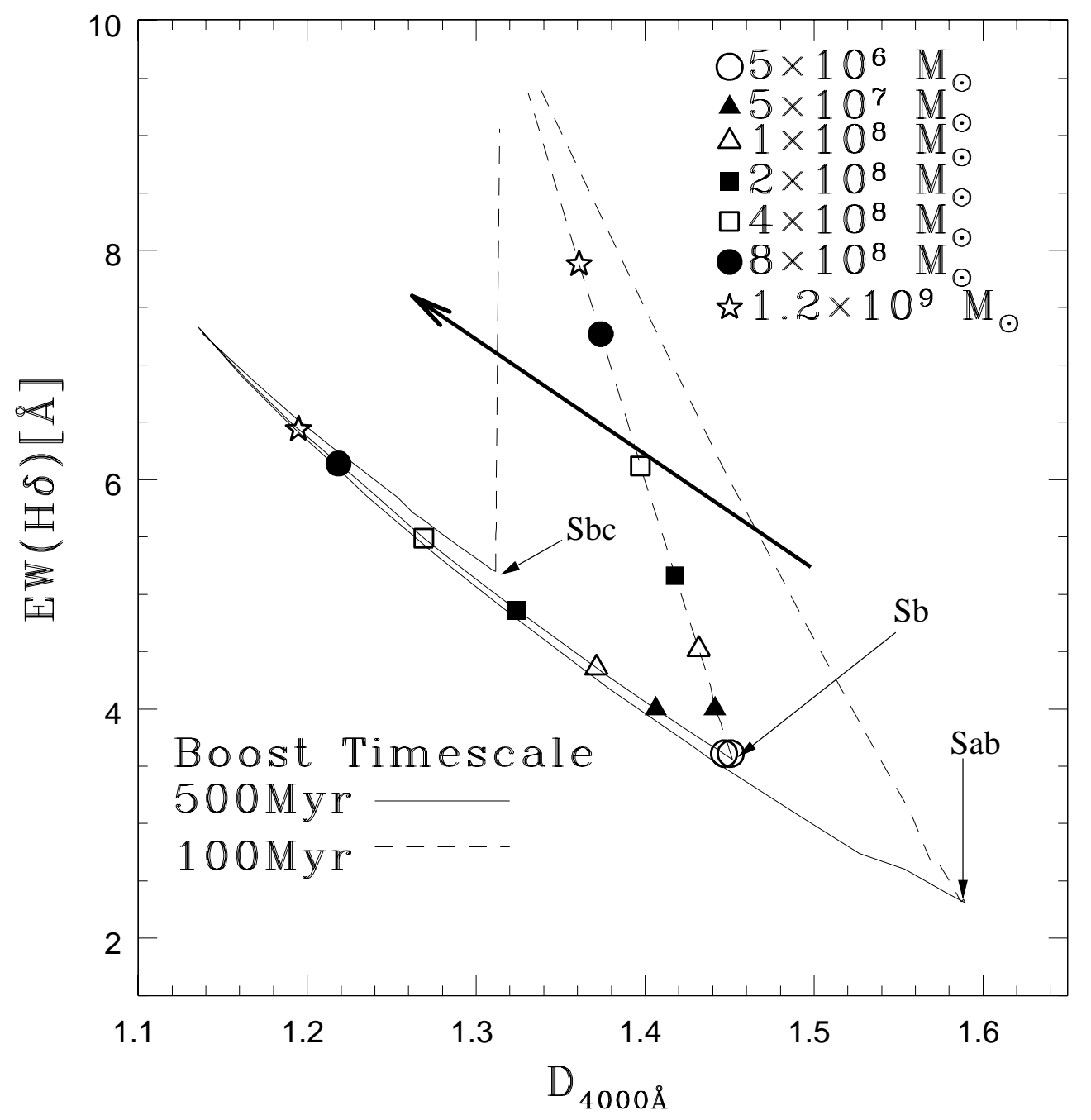

Fig. 6.- Model tracks for the "underlying population + boost" EPS models. Each track represents the evolution of $D_{4000}$ and $E W_{\text {mod }}\left(H \delta_{a b s}\right)$ with boost strength for a given Hubble type (as parameterized by $b$ ) and boost timescale. The symbols represent different masses formed in the boost in addition to that formed in the underlying population over the past 0.5 Gyr. We also overlay the boost vector connecting the median values of the spectral indices for the most symmetric third, to the median values for the most lopsided third. For additional reference, we plot tracks for galaxies of Hubble type Sab, Sb, and Sbc. 


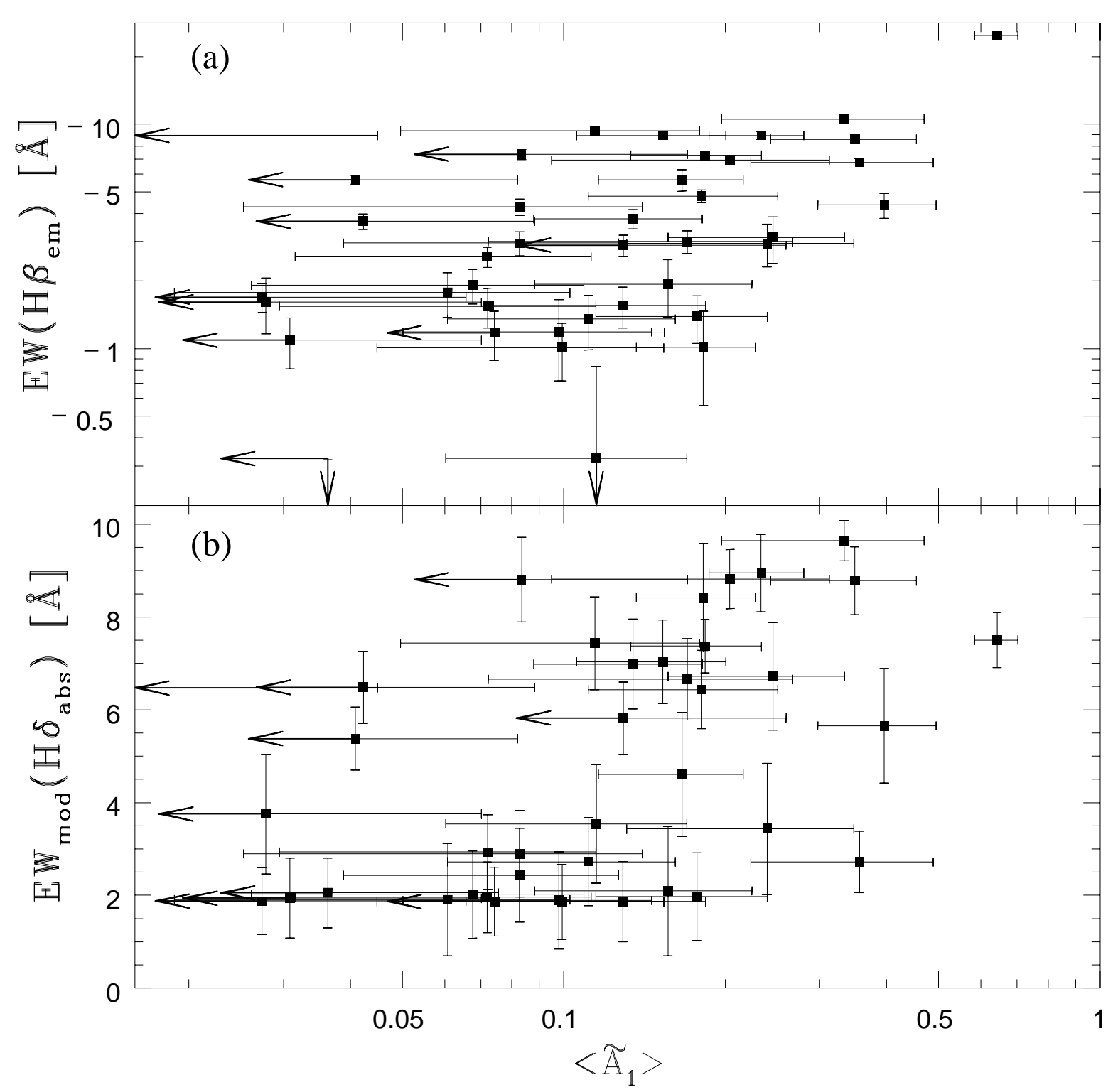

Fig. 7.- Measures of the current SFR and recent SFH in the nucleus vs. $\left\langle\tilde{A}_{1}\right\rangle$ : (a) $E W\left(H \beta_{e m}\right)$ as measured from the decomposed "emission" spectrum of each galaxy. (b) $E W_{\text {mod }}\left(H \delta_{a b s}\right)$ as measured from our best fit stellar template model to the "absorption" spectrum of the galaxy $I_{\text {model }}^{\text {best }}(\lambda)$. Arrows on data points indicate upper limits. 
Table 1. Sample: Summary of RC3 Parameters

\begin{tabular}{lcccll}
\hline \hline object name & $\mathrm{T}$ & $\begin{array}{c}\mathrm{D}^{\mathrm{a}} \\
\left({ }^{\prime}\right)\end{array}$ & $\begin{array}{c}\mathrm{v}_{r e c} \\
\left(k m s^{-1}\right)\end{array}$ & $\mathrm{d} / \mathrm{D}^{\mathrm{b}}$ & $\begin{array}{c}L_{B} \\
\left(10^{21} h^{-2} W H z^{-1}\right)\end{array}$ \\
\hline A 1219+41 & 3.0 & 1.19 & 6927 & 0.85 & $7.52 \pm 1.03$ \\
IC 0520 & 2.0 & 1.95 & 3528 & 0.79 & $6.06 \pm 0.90$ \\
IC 0900 & 4.0 & 1.62 & 7067 & 0.65 & $9.30 \pm 0.89$ \\
IC 1269 & 4.0 & 1.70 & 6116 & 0.74 & $8.21 \pm 1.57$ \\
NGC 2347 & 3.0 & 1.78 & 4422 & 0.71 & $5.32 \pm 0.79$ \\
NGC 2582 & 2.0 & 1.23 & 4439 & 1.00 & $2.79 \pm 0.35$ \\
NGC 2744 & 2.0 & 1.66 & 3428 & 0.65 & $1.62 \pm 0.33$ \\
NGC 2916 & 3.0 & 2.45 & 3730 & 0.68 & $5.50 \pm 0.76$ \\
NGC 3066 & 4.0 & 1.10 & 2049 & 0.89 & $0.78 \pm 0.12$ \\
NGC 3162 & 4.0 & 3.02 & 1298 & 0.83 & $1.41 \pm 0.18$ \\
NGC 3177 & 3.0 & 1.44 & 1302 & 0.81 & $0.46 \pm 0.06$ \\
NGC 3310 & 4.0 & 3.09 & 0980 & 0.78 & $1.84 \pm 0.18$ \\
NGC 3353 & 3.0 & 1.35 & 0944 & 0.71 & $0.22 \pm 0.03$ \\
NGC 3681 & 4.0 & 2.51 & 1239 & 0.79 & $1.49 \pm 0.48$ \\
NGC 3684 & 4.0 & 3.09 & 1163 & 0.69 & $1.57 \pm 0.50$ \\
NGC 3897 & 4.0 & 1.95 & 6411 & 1.00 & $7.93 \pm 1.17$ \\
NGC 3928 & 3.0 & 1.51 & 0982 & 1.00 & $0.24 \pm 0.03$ \\
NGC 3963 & 4.0 & 2.75 & 3186 & 0.91 & $5.23 \pm 1.67$ \\
NGC 4017 & 4.0 & 1.78 & 3454 & 0.78 & $3.72 \pm 1.18$ \\
NGC 4041 & 4.0 & 2.69 & 1234 & 0.93 & $1.27 \pm 0.16$ \\
NGC 4351 & 2.0 & 2.00 & 2310 & 0.68 & $1.79 \pm 0.26$ \\
NGC 4412 & 3.0 & 1.41 & 2294 & 0.89 & $1.43 \pm 0.21$ \\
NGC 4430 & 3.0 & 2.29 & 1443 & 0.89 & $0.85 \pm 0.13$ \\
NGC 4595 & 3.0 & 1.74 & 0633 & 0.65 & $0.12 \pm 0.18$ \\
NGC 4639 & 4.0 & 2.75 & 1010 & 0.68 & $0.59 \pm 0.05$ \\
NGC 4814 & 3.0 & 3.09 & 2513 & 0.74 & $2.48 \pm 0.50$ \\
NGC 4911 & 4.0 & 1.45 & 7970 & 0.91 & $12.04 \pm 1.53$ \\
NGC 5218 & 3.0 & 1.82 & 2807 & 0.69 & $2.31 \pm 1.02$ \\
NGC 5614 & 2.0 & 2.45 & 3892 & 0.83 & $7.50 \pm 0.95$ \\
NGC 5653 & 3.0 & 1.74 & 3567 & 0.74 & $4.63 \pm 0.59$ \\
NGC 5713 & 4.0 & 2.75 & 1883 & 0.89 & $3.32 \pm 0.49$ \\
NGC 5915 & 4.0 & 1.82 & 5570 & 1.00 & $2.04 \pm 0.26$ \\
NGC 5923 & 2.0 & 1.74 & 2291 & 0.72 & $5.20 \pm 0.88$ \\
NGC 5936 & 3.0 & 1.45 & 4004 & 0.89 & $4.76 \pm 0.60$ \\
& & & & & \\
NGC & &
\end{tabular}


Table 1 - Continued

\begin{tabular}{lcccll}
\hline \hline object name & $\mathrm{T}$ & $\begin{array}{c}\mathrm{D}^{\mathrm{a}} \\
\left({ }^{\prime}\right)\end{array}$ & $\begin{array}{c}\mathrm{v}_{\text {rec }} \\
\left(k m s^{-1}\right)\end{array}$ & $\mathrm{d} / \mathrm{D}^{\mathrm{b}}$ & $\begin{array}{c}L_{B} \\
\left(10^{21} h^{-2} W H z^{-1}\right)\end{array}$ \\
\hline NGC 6012 & 2.0 & 2.09 & 1854 & 0.72 & $1.69 \pm 0.21$ \\
NGC 6195 & 3.0 & 1.55 & 9000 & 0.69 & $12.89 \pm 2.19$ \\
NGC 6574 & 4.0 & 1.41 & 2282 & 0.78 & $2.02 \pm 0.26$ \\
NGC 6711 & 4.0 & 1.35 & 4671 & 0.93 & $3.63 \pm 0.50$ \\
NGC 6824 & 3.0 & 1.70 & 3337 & 0.69 & $3.58 \pm 0.72$ \\
NGC 7177 & 3.0 & 3.10 & 1150 & 0.64 & $0.99 \pm 0.12$ \\
\hline
\end{tabular}

${ }^{a}$ the diameter of the galaxy out to the isophote of surface brightness $25 \mathrm{mag} / \mathrm{asec}^{2} ; D=0.1^{\prime} \times 10^{D_{25}}$

b the ratio of the minor to major axis as defined in the RC3 catalog; $d / D=10^{-R_{25}}$ 
Table 2. Analysis Results

\begin{tabular}{|c|c|c|c|c|c|c|c|}
\hline object name & $\begin{array}{l}\mathrm{R}_{\exp } \\
\left({ }^{\prime \prime}\right)\end{array}$ & $\mathrm{R}_{\max } / \mathrm{R}_{\exp }$ & $<\tilde{\mathrm{A}}_{1}>$ & $\begin{array}{c}E W\left(H \beta_{e m}\right) \\
(\AA)\end{array}$ & $\begin{array}{c}E W_{\text {mod }}\left(H \delta_{a b s}\right) \\
(\AA)\end{array}$ & $C_{A 0 V}$ & $D_{4000}$ \\
\hline A $1219+41$ & 19.7 & 3.15 & $0.083 \pm 0.057$ & $-3.7 \pm 0.3$ & $8.1 \pm 0.6$ & 0.34 & $1.18 \pm 0.01$ \\
\hline IC 0520 & 19.8 & 3.82 & $0.031 \pm 0.039$ & $-2.3 \pm 0.2$ & $4.4 \pm 0.7$ & 0.16 & $1.72 \pm 0.01$ \\
\hline IC 0900 & 11.6 & 6.54 & $0.156 \pm 0.068$ & $-6.1 \pm 0.3$ & $8.0 \pm 0.7$ & 0.38 & $1.32 \pm 0.01$ \\
\hline IC 1269 & 13.0 & 2.93 & $0.239 \pm 0.108$ & $-6.3 \pm 0.5$ & $6.9 \pm 0.9$ & 0.32 & $1.34 \pm 0.02$ \\
\hline NGC 2347 & 10.6 & 5.34 & $0.083 \pm 0.044$ & $-4.9 \pm 0.3$ & $6.5 \pm 0.7$ & 0.32 & $1.12 \pm 0.01$ \\
\hline NGC 2582 & 20.0 & 2.82 & $0.061 \pm 0.042$ & $-2.7 \pm 0.3$ & $5.6 \pm 0.9$ & 0.24 & $1.59 \pm 0.02$ \\
\hline NGC 2744 & 10.8 & 4.28 & $0.233 \pm 0.047$ & $-5.4 \pm 0.4$ & $9.6 \pm 0.8$ & 0.60 & $1.32 \pm 0.01$ \\
\hline NGC 2916 & 23.0 & 3.29 & $0.177 \pm 0.062$ & $-4.3 \pm 0.2$ & $7.0 \pm 0.6$ & 0.36 & $1.47 \pm 0.01$ \\
\hline NGC 3066 & 13.7 & 3.40 & $0.356 \pm 0.132$ & $-6.4 \pm 0.2$ & $7.0 \pm 0.6$ & 0.37 & $1.05 \pm 0.01$ \\
\hline NGC 3162 & 26.0 & 2.91 & $0.181 \pm 0.070$ & $-6.8 \pm 0.3$ & $8.6 \pm 0.7$ & 0.52 & $1.42 \pm 0.01$ \\
\hline NGC 3177 & 8.6 & 6.58 & $0.183 \pm 0.050$ & $-6.6 \pm 0.2$ & $7.2 \pm 0.5$ & 0.37 & $1.213 \pm 0.005$ \\
\hline NGC 3310 & 9.92 & 7.63 & $0.334 \pm 0.137$ & $-20.1 \pm 0.2$ & $8.0 \pm 0.4$ & 0.45 & $0.963 \pm 0.002$ \\
\hline NGC 3353 & 12.1 & 4.23 & $0.643 \pm 0.059$ & $-22.2 \pm 0.2$ & $6.8 \pm 0.6$ & 0.38 & $0.883 \pm 0.004$ \\
\hline NGC 3681 & 24.9 & 3.03 & $0.027 \pm 0.038$ & $-2.6 \pm 0.2$ & $3.7 \pm 0.7$ & 0.12 & $1.18 \pm 0.01$ \\
\hline NGC 3684 & 16.8 & 4.51 & $0.042 \pm 0.046$ & $-5.1 \pm 0.3$ & $6.6 \pm 0.7$ & 0.34 & $1.23 \pm 0.01$ \\
\hline NGC 3897 & 12.6 & 4.49 & $0.099 \pm 0.054$ & $-3.8 \pm 0.3$ & $5.6 \pm 0.9$ & 0.25 & $1.12 \pm 0.01$ \\
\hline NGC 3928 & 18.7 & 4.03 & $0.009 \pm 0.036$ & $-6.6 \pm 0.3$ & $6.7 \pm 0.7$ & 0.35 & $1.42 \pm 0.01$ \\
\hline NGC 3963 & 25.4 & 2.98 & $0.135 \pm 0.047$ & $-3.4 \pm 0.2$ & $8.0 \pm 0.6$ & 0.34 & $1.19 \pm 0.01$ \\
\hline NGC 4017 & 16.0 & 3.89 & $0.083 \pm 0.087$ & $-7.0 \pm 0.3$ & $8.3 \pm 0.7$ & 0.48 & $1.15 \pm 0.01$ \\
\hline NGC 4041 & 10.8 & 6.97 & $0.041 \pm 0.041$ & $-6.4 \pm 0.2$ & $6.8 \pm 0.5$ & 0.35 & $1.20 \pm 0.005$ \\
\hline NGC 4351 & 18.1 & 4.18 & $0.153 \pm 0.047$ & $-4.2 \pm 0.3$ & $7.3 \pm 0.7$ & 0.42 & $0.98 \pm 0.01$ \\
\hline NGC 4412 & 23.7 & 2.90 & $0.396 \pm 0.098$ & $-6.0 \pm 0.2$ & $7.8 \pm 0.6$ & 0.40 & $1.26 \pm 0.01$ \\
\hline NGC 4430 & 28.3 & 2.67 & $0.245 \pm 0.089$ & $-5.1 \pm 0.3$ & $7.4 \pm 0.8$ & 0.35 & $1.29 \pm 0.01$ \\
\hline NGC 4595 & 16.3 & 3.82 & $0.170 \pm 0.097$ & $-4.2 \pm 0.2$ & $7.9 \pm 0.6$ & 0.46 & $1.003 \pm 0.005$ \\
\hline NGC 4639 & 23.4 & 3.24 & $0.072 \pm 0.043$ & $-2.9 \pm 0.2$ & $4.9 \pm 0.6$ & 0.15 & $1.52 \pm 0.01$ \\
\hline NGC 4814 & 13.5 & 5.58 & $0.068 \pm 0.041$ & $-3.2 \pm 0.3$ & $5.2 \pm 0.7$ & 0.17 & $1.50 \pm 0.01$ \\
\hline NGC 4911 & 14.5 & 3.54 & $0.129 \pm 0.055$ & $-3.2 \pm 0.3$ & $6.5 \pm 0.8$ & 0.27 & $1.38 \pm 0.01$ \\
\hline NGC 5218 & 8.7 & 4.86 & $0.182 \pm 0.046$ & $-1.6 \pm 0.2$ & $7.6 \pm 0.6$ & 0.29 & $1.37 \pm 0.01$ \\
\hline NGC 5614 & 11.9 & 4.76 & $0.074 \pm 0.080$ & $-1.7 \pm 0.3$ & $3.9 \pm 0.8$ & 0.10 & $1.71 \pm 0.01$ \\
\hline NGC 5653 & 6.8 & 5.63 & $0.129 \pm 0.131$ & $-5.2 \pm 0.2$ & $8.0 \pm 0.5$ & 0.36 & $1.343 \pm 0.005$ \\
\hline NGC 5713 & 14.6 & 4.27 & $0.349 \pm 0.106$ & $-6.2 \pm 0.2$ & $9.6 \pm 0.5$ & 0.48 & $1.113 \pm 0.003$ \\
\hline NGC 5915 & 5.0 & 3.48 & $0.204 \pm 0.109$ & $-11.5 \pm 0.2$ & $8.7 \pm 0.6$ & 0.51 & $1.146 \pm 0.005$ \\
\hline NGC 5923 & 15.2 & 2.52 & $0.115 \pm 0.055$ & $-2.6 \pm 0.4$ & $6.2 \pm 0.9$ & 0.23 & $1.44 \pm 0.02$ \\
\hline NGC 5936 & 11.7 & 3.61 & $0.114 \pm 0.065$ & $-7.6 \pm 0.2$ & $8.3 \pm 0.6$ & 0.44 & $1.218 \pm 0.005$ \\
\hline
\end{tabular}


Table 2-Continued

\begin{tabular}{llllllll}
\hline \hline object name & $\begin{array}{c}\mathrm{R}_{\text {exp }} \\
\left({ }^{\prime \prime}\right)\end{array}$ & $\mathrm{R}_{\text {max }} / \mathrm{R}_{\text {exp }}$ & \multicolumn{1}{c}{$<\tilde{\mathrm{A}}_{1}>$} & $\begin{array}{c}E W\left(H \beta_{\text {em }}\right) \\
(\AA)\end{array}$ & $\begin{array}{c}E W_{\text {mod }}\left(H \delta_{a b s}\right) \\
(\AA)\end{array}$ & $C_{A 0 V}$ & $D_{4000}$ \\
\hline NGC 6012 & 12.2 & 3.46 & $0.028 \pm 0.042$ & $-3.5 \pm 0.3$ & $4.6 \pm 0.9$ & 0.15 & $1.45 \pm 0.02$ \\
NGC 6195 & 11.2 & 4.17 & $0.098 \pm 0.048$ & $-3.6 \pm 0.4$ & $6.1 \pm 0.9$ & 0.25 & $1.30 \pm 0.02$ \\
NGC 6574 & 8.6 & 4.46 & $0.111 \pm 0.050$ & $-5.6 \pm 0.2$ & $7.2 \pm 0.5$ & 0.31 & $1.43 \pm 0.01$ \\
NGC 6711 & 9.2 & 3.13 & $0.166 \pm 0.050$ & $-6.2 \pm 0.3$ & $7.5 \pm 0.8$ & 0.38 & $1.25 \pm 0.01$ \\
NGC 6824 & 14.5 & 4.64 & $0.068 \pm 0.1$ & $-2.4 \pm 0.2$ & $6.5 \pm 0.5$ & 0.24 & $1.50 \pm 0.01$ \\
NGC 7177 & 10.9 & 5.66 & $0.140 \pm 0.1$ & $-1.7 \pm 0.2$ & $4.1 \pm 0.6$ & 0.12 & $1.59 \pm 0.01$ \\
& & & & & & & \\
\hline
\end{tabular}

${ }^{a}$ The fourth column is the averages of two images 